\title{
Projective curves with maximal regularity and applications to syzygies and surfaces
}

\author{
Brodmann, M ; Schenzel, $\mathrm{P}$
}

\begin{abstract}
The authors of this paper show that the union of a projective curve with one of its extremal secant lines satisfies the linear general position principle for hyperplane sections. They use it to improve the approximation of the Betti numbers of curves
\end{abstract}

DOI: https://doi.org/10.1007/s00229-011-0426-0

Posted at the Zurich Open Repository and Archive, University of Zurich

ZORA URL: https://doi.org/10.5167/uzh-53623

Journal Article

Accepted Version

Originally published at:

Brodmann, M; Schenzel, P (2011). Projective curves with maximal regularity and applications to syzygies and surfaces. Manuscripta Mathematica, 135(3-4):469-495.

DOI: https://doi.org/10.1007/s00229-011-0426-0 


\title{
PROJECTIVE CURVES WITH MAXIMAL REGULARITY AND APPLICATIONS TO SYZYGIES AND SURFACES
}

\author{
MARKUS BRODMANN PETER SCHENZEL
}

\begin{abstract}
We first show that the union of a projective curve with one of its extremal secant lines satisfies the linear general position principle for hyperplane sections. We use this to give an improved approximation of the Betti numbers of curves $\mathcal{C} \subset \mathbb{P}_{K}^{r}$ of maximal regularity with $\operatorname{deg} \mathcal{C} \leq 2 r-3$. In particular we specify the number and degrees of generators of the vanishing ideal of such curves. We apply these results to study surfaces $X \subset \mathbb{P}_{K}^{r}$ whose generic hyperplane section is a curve of maximal regularity. We first give a criterion for "an early decent of the Hartshorne-Rao function" of such surfaces. We use this criterion to give a lower bound on the degree for a class of these surfaces. Then, we study surfaces $X \subset \mathbb{P}_{K}^{r}$ for which $h^{1}\left(\mathbb{P}_{K}^{r}, \mathcal{I}_{X}(1)\right)$ takes a value close to the possible maximum $\operatorname{deg} X-r+1$. We give a lower bound on the degree of such surfaces. We illustrate our results by a number of examples, computed by means of SiNGULAR, which show a rich variety of occuring phenomena.
\end{abstract}

\section{INTRODUCTION}

The aim of this paper is to study projective curves of maximal regularity and applications to certain types of projective surfaces. Much emphasis will be given to the computation of examples which illustrate the proved results.

We begin with an investigation on curves of maximal regularity and their extremal secant lines. To be more precise, let $\mathcal{C} \subset \mathbb{P}_{K}^{r}$ be a non-degenerate irreducible projective curve in projective $r$-space over the algebraically closed field $K$, with $r \geq 3$. Let $d:=\operatorname{deg} \mathcal{C}$ denote the degree of $\mathcal{C}$. Assume that $d>r+1$ and that $\mathcal{C}$ is of maximal regularity, so that

$$
\operatorname{reg} \mathcal{C}=d-r+2 .
$$

(Keep in mind that according to $[\mathrm{GLP}]$ we always have reg $\mathcal{C} \leq d-r+2$.) In this extremal situation it is known that $\mathcal{C}$ is smooth and rational and has a $(d-r+2)$-secant line $\mathbb{L}$. We fix such an extremal secant line $\mathbb{L}=\mathbb{P}_{K}^{1} \subset \mathbb{P}_{K}^{r}$, so that

$$
\lambda\left(\mathcal{O}_{\mathcal{C} \cap \mathbb{L}}\right)=d-r+2 .
$$

In [BS2] we have shown that the scheme $\mathcal{C} \cup \mathbb{L} \subset \mathbb{P}_{K}^{r}$ plays a crucial rôle for the understanding of the curve $\mathcal{C}$ in particular its syzygetic behaviour. We first take up this idea and prove that in some sense the scheme $\mathcal{C} \cup \mathbb{L}$ behaves like an irreducible curve, namely (cf. Corollary 2.6):

2000 Mathematics Subject Classification. Primary: 14H45; 14Q10 ; Secondary: 14J25.

Key words and phrases. curves; surfaces; maximal regularity.

The second named author was partially supported by Swiss National Science Foundation (Project No. $20-111762)$. 
Theorem 1.1. Let $r>3$. Then the generic hyperplane section $(\mathcal{C} \cup \mathbb{L}) \cap \mathbb{H} \subset \mathbb{H}=\mathbb{P}_{K}^{r-1}$ of $\mathcal{C} \cup \mathbb{L}$ is a reduced scheme of $d+1$ points in linearly general position.

Now, on use of the $N_{p}$-Theorem of Green and Lazarsfeld [13] we then may approximate the Betti numbers of the homogeneous vanishing ideal $I \subset S:=K\left[x_{0}, \ldots, x_{r}\right]$ of the curve $\mathcal{C}$, provided $d$ is not to large (cf. Theorem 3.3)

Theorem 1.2. Let $r>3$ and $r+2 \leq d \leq 2 r-3$. Then, for all $i \in\{1, \ldots, r\}$ we have

$$
\operatorname{Tor}_{i}^{S}(K, S / I) \simeq K^{u_{i}}(-i-1) \oplus K^{v_{i}}(-i-2) \oplus K^{\left(\begin{array}{c}
r-1 \\
i-1
\end{array}\right)}(-i-d+r-1)
$$

with $v_{i}=0$ for $1 \leq i \leq 2 r-d-2$ and $u_{1}=\left(\begin{array}{c}r+1 \\ 2\end{array}\right)-d-1$.

In particular we get (cf. Corollary $3.3(\mathrm{~b})$ ):

Corollary 1.3. Under the hypothesis of 1.2 the vanishing ideal $I \subset S$ of $\mathcal{C}$ is minimally generated by $\left(\begin{array}{c}r+1 \\ 2\end{array}\right)-d-1$ quadrics and one form of degree $d-r+2$.

In the second part of our paper we apply these results to certain surfaces. Our first aim is to study a fairly technical issue concerning non-degenerate irreducible projective surfaces $X \subset \mathbb{P}_{K}^{r}$ of degree $d \leq 2 r-4$. Namely, we consider the "least place at which the Hartshorne-Rao function $n \mapsto h^{1}\left(\mathbb{P}_{K}^{r}, \mathcal{I}_{X}(n)\right)$ of $X$ definitively begins to decent", that is the invariant

$$
\delta(X):=\inf \left\{m \in \mathbb{Z} \mid h^{1}\left(\mathbb{P}_{K}^{r}, \mathcal{I}_{X}(n)\right) \leq \max \left\{h^{1}\left(\mathbb{P}_{K}^{r}, \mathcal{I}_{X}(n-1)\right)-1,0\right\} \text { for all } m>n\right\} .
$$

It follows by Mumford's Lemma on the decent of the Hartshorne-Rao function (cf. [19]) that $\delta(X) \leq d-r+2$. We are interested to find criteria which guarantee that this inequality is strict. One sufficient condition surely would be that $X$ satisfies the Regularity Conjecture of Eisenbud and Goto [10], that is the inequality reg $X \leq d-r+3$ (cf. Lemma 4.5). Again by Mumford's Lemma one has $\delta(X)<d-r+2$ if the generic hyperplane section of $X$ is not of maximal regularity (cf. Lemma 4.5). We shall prove another criterion, namely (cf. Corollary 4.6):

Corollary 1.4. Let $r>4$ and $r<d \leq 2 r-4$. Then $\delta(X) \leq h^{1}\left(\mathbb{P}_{K}^{r}, \mathcal{I}_{X}(1)\right)+h^{1}\left(X, \mathcal{O}_{X}\right)$.

Next we study surfaces of maximal sectional regularity, that is projective surfaces $X \subset \mathbb{P}_{K}^{r}$ whose generic hyperplane section is a curve of maximal regularity. For such surfaces reg $X$ takes at least the value $d-r+3$, the maximally possible value conjectured by Eisenbud and Gôto. Neverless there are surfaces $X$ satisfying reg $X=d-r+3$ which are not of maximal sectional regularity (cf. Remark 5.4 (B)). Surfaces of maximal sectional regularity have "a lot" of extremal secant lines in the sense of Bertin [1], (cf. Proposition 5.5 and Corollaries 5.6, 5.7). We prove the following bound on the degree $d$ of these surfaces (cf. Theorem 5.10).

Theorem 1.5. Assume that $4<r<d$ and that $X$ is of maximal sectional regularity and of arithmetic depth one. Then $\delta(X) \leq d-r+1$ implies that $d>2 r-5$.

It follows in particular (cf. Corollary 5.13):

Corollary 1.6. Let $4<r<d$, assume that $X \subset \mathbb{P}_{K}^{r}$ is Cohen-Macaulay, of maximal sectional regularity and of arithmetic depth one. Then $d>2 r-5$. 
It is well known that $h:=h^{1}\left(\mathbb{P}_{K}^{r}, \mathcal{I}_{X}(1)\right) \leq d-r+1$. In Section 6 we study surfaces $X$ with "large $h$ ", that is with $d-r-1 \leq h \leq d-r+1$. Using the concept of maximal projecting surface $Y \subset \mathbb{P}_{K}^{r+h}$ for $X$ (cf. Reminder 5.12) and the description of the possible types of surfaces $Z \subset \mathbb{P}_{K}^{s}$ of degree $s+1$ given in [4] we prove (see Theorem 6.3 for more details):

Theorem 1.7. Let $4<r<d$. Then

(a) If $h=d-r+1$, the surface $X$ is smooth and rational.

(b) If $h=d-r$, then either

(i) $X$ is Cohen-Macaulay and the non-normal locus of $X$ is a straight line, or

(ii) $X$ contains a single non-Cohen-Macaulay point and is normal outside this point.

(c) If $h=d-r-1$, we distinguish five different cases according to the type of the maximal projective surface $Y \subset \mathbb{P}_{K}^{r+h}$ for $X$.

An important issue of our paper are the examples contained in Section 7, which illustrate our results. These examples were computed by using the computer algebra system Singular (cf. [14]).

Acknowledgement. The main results of the present paper were found during a stay of the authors at the "Mathematisches Forschungsinstitiut Oberwolfach" in the framework of the "Research in Pairs" scheme. The authors express their gratitude toward this institution and the DFG for financial support and hospitality.

\section{Generic Hyperplane Sections of $\mathcal{C} \cup \mathbb{L}$}

Here let $\mathcal{C} \subset \mathbb{P}_{K}^{r}$ be a non-degenerate irreducible projective curve in projective $r$-space of maximal regularity $\operatorname{reg} \mathcal{C}=d-r+2$. Then $\mathcal{C}$ has a $(d-r+2)$-secant line $\mathbb{L}$. In this section we show that generic hyperplane sections of the union $\mathcal{C} \cup \mathbb{L}$ are reduced schemes of points in linearly general position.

Notation 2.1. Let $\pi: \mathbb{P}_{K}^{r} \backslash \mathbb{L} \rightarrow \mathbb{P}_{K}^{r-2}$ denote a linear projection with center $\mathbb{L}$ and let

$$
\mathcal{C}^{\prime}:=\overline{\pi(\mathcal{C} \backslash \mathbb{L})} \subset \mathbb{P}_{K}^{r-2}
$$

be the closed image of $\mathcal{C} \backslash \mathbb{L}$ under $\pi$. Observe that $\mathcal{C}^{\prime} \subset \mathbb{P}_{K}^{r-2}$ is a reduced irreducible and non-degenerate curve.

In characteristic 0, statement (a) of the following Lemma 2.2 has an important extension for arbitrary varieties which admit an extremal secant line (cf. [2, Corollary 4.2]).

Lemma 2.2. Let $r>3$. Then:

(a) $\mathcal{C}^{\prime} \subset \mathbb{P}_{K}^{r-2}$ is a rational normal curve.

(b) The map $\nu=\pi \uparrow: \mathcal{C} \backslash \mathbb{L} \rightarrow \mathbb{P}_{K}^{r-2}$ is an immersion.

Proof. (a): Let $d^{\prime}:=\operatorname{deg} \mathcal{C}^{\prime}$. As $\mathcal{C}^{\prime} \subset \mathbb{P}_{K}^{r-2}$ is non-degenerate, it suffices to show that $d^{\prime} \leq r-2$. But this is well known (cf. [2, Corollary 4.2] for example).

(b): It suffices to show that $\nu: \mathcal{C} \backslash \mathbb{L} \rightarrow \mathcal{C}^{\prime}$ is an immersion. As $\mathcal{C} \backslash \mathbb{L}$ is affine and $\mathcal{C}^{\prime} \simeq \mathbb{P}_{K}^{1}$ it suffices to show that $\nu: \mathcal{C} \backslash \mathbb{L} \rightarrow \mathcal{C}^{\prime}$ is injective. Assume to the contrary that there are two points $p_{1}, p_{1}^{\prime} \in \mathcal{C} \backslash \mathbb{L}$ such that $p_{1} \neq p_{1}^{\prime}$ but $\nu\left(p_{1}\right)=\nu\left(p_{1}^{\prime}\right)=: q_{1} \in \mathbb{P}_{K}^{r-2}$. 
Choose pairwise different points $q_{2}, \ldots, q_{r-2} \in \nu(\mathcal{C} \backslash \mathbb{L}) \backslash\left\{q_{1}\right\}$. As $\mathcal{C}^{\prime} \subset \mathbb{P}_{K}^{r-2}$ is a rational normal curve by statement (a) we see that

$$
\mathbb{H}^{\prime}:=\left\langle q_{1}, \ldots, q_{r-2}\right\rangle \subset \mathbb{P}_{K}^{r-2}
$$

is a hyperplane. Consider the hyperplane

$$
\mathbb{H}:=\nu^{-1}\left(\mathbb{H}^{\prime}\right)=\nu^{-1}\left(\mathbb{H}^{\prime}\right) \cup \mathbb{L} \text {. }
$$

For each $i \in\{2, \ldots, r-2\}$ let $p_{i} \in \mathcal{C} \backslash \mathbb{L}$ be such that $\nu\left(p_{i}\right)=q_{i}$, so that $p_{1}^{\prime}, p_{1}, p_{2}, \ldots, p_{r-2} \in$ $(\mathcal{C} \cap \mathbb{H}) \backslash \mathbb{L}$ are pairwise different points. The inequalities used in the proof of statement (a) show that $\#\left((\mathcal{C} \cap \mathbb{H})_{\text {red }} \backslash \mathbb{L}\right) \leq r-2$. So, we have a contradiction.

Lemma 2.3. Let $r>3$ and $p_{1}, \ldots, p_{r-1} \in \mathcal{C} \backslash \mathbb{L}$ be pairwise different points. Then $\left\langle p_{1}, \ldots, p_{r-1}\right\rangle \subset \mathbb{P}_{K}^{r}$ is of dimension $r-2$ and disjoint to $\mathbb{L}$.

Proof. Let $\mathbb{K}:=\left\langle p_{1}, \ldots, p_{r-1}\right\rangle$. By Lemma 2.2 and the notation of 2.1 the $r-1$ points $\pi\left(p_{1}\right), \ldots, \pi\left(p_{r-1}\right) \in \mathcal{C}^{\prime}$ are pairwise different. As $\mathcal{C}^{\prime} \subset \mathbb{P}_{K}^{r-2}$ is a rational normal curve we get $\left\langle\pi\left(p_{1}\right), \ldots, \pi\left(p_{r-2}\right)\right\rangle=\mathbb{P}_{K}^{r-2}$. Therefore $\pi(\mathbb{K} \backslash \mathbb{L})=\langle\pi(\mathbb{K} \backslash \mathbb{L})\rangle \supseteqq \mathbb{P}_{K}^{r-2}$, whence

$$
r-2 \leq \operatorname{dim} \pi(\mathbb{K} \backslash \mathbb{L}) \leq \operatorname{dim} \mathbb{K} \leq r-2,
$$

thus $\operatorname{dim} \pi(\mathbb{K} \backslash \mathbb{L})=\operatorname{dim} \mathbb{K}=r-2$. The first inequality implies in particular that $\mathbb{K} \cap \mathbb{L}=$ $\emptyset$.

Reminder 2.4. (A) Let $s, d \in \mathbb{N}$ with $s<d$ and let $p_{1}, \ldots, p_{d} \in \mathbb{P}_{K}^{s}$ be pairwise different points. Let $P=\left\{p_{1}, \ldots, p_{d}\right\}$. We say that $p_{1}, \ldots, p_{d}$ are in linearly general position if for all subsets $Q \subseteq P$ with $\# Q=s+1$ it follows that $\langle A\rangle=\mathbb{P}_{K}^{s}$. This is equivalent to say that for all $Q \subseteq P$ we have $\operatorname{dim}\langle Q\rangle=\max \{\# Q-1, s\}$.

(B) Let $p_{d+1} \in \mathbb{P}_{K}^{s}$ and assume that $p_{1}, \ldots, p_{d}$ are in linearly general position. Then $p_{1}, \ldots, p_{d}, p_{d+1}$ are in linearly general position if and only if for each set $Q \subseteq\left\{p_{1}, \ldots, p_{d}\right\}$ of cardinality $s$ we have $p_{d+1} \notin\langle Q\rangle$.

Proposition 2.5. Let $r>3$ and let $\mathbb{H} \subset \mathbb{P}_{K}^{r}$ be a hyperplane such that $\mathbb{H} \cap(\mathcal{C} \cap \mathbb{L})=\emptyset$ and such that $\mathcal{C} \cap \mathbb{H} \subset \mathbb{H}$ is a reduced subscheme of d points in linearly general position. Then $(\mathcal{C} \cup \mathbb{L}) \cap \mathbb{H} \subset \mathbb{H}$ is a reduced subscheme of $d+1$ points in linearly general position.

Proof. We write $|\mathcal{C} \cap \mathbb{H}|=\left\{p_{1}, \ldots, p_{d}\right\}$ with pairwise different points $p_{1}, \ldots, p_{d}$ and $\left\{p_{d+1}\right\}=\mathbb{H} \cap \mathbb{L}$. As $\mathbb{H} \cap(\mathcal{C} \cap \mathbb{L})=\emptyset$, the points $p_{1}, \ldots, p_{d}, p_{d+1}$ are pairwise different.

As $\operatorname{deg} \mathcal{C} \cup \mathbb{L}=d+1$ and

$$
|\mathbb{H} \cap(\mathcal{C} \cup \mathbb{L})| \supseteq\left\{p_{1}, \ldots, p_{d}, p_{d+1}\right\}
$$

it follows that $\mathbb{H} \cap(\mathcal{C} \cup \mathbb{L})$ is a reduced scheme of $d+1$ points. It remains to show that the points $p_{1}, \ldots, p_{d}, p_{d+1} \in \mathbb{H}=\mathbb{P}_{K}^{r-1}$ are in linearly general position. This follows by Lemma 2.3 and Reminder $2.4(\mathrm{~B})$.

Corollary 2.6. Let $r>3$. For a generic hyperplane $\mathbb{H} \subset \mathbb{P}_{K}^{r}$ the subscheme $(\mathcal{C} \cap \mathbb{L}) \cap \mathbb{H} \subseteq$ $\mathbb{H}=\mathbb{P}_{K}^{r-1}$ is a reduced subscheme of $d+1$ points in linearly general position.

Proof. This is immediate from Proposition 2.5. 


\section{Estimates of Betti Numbers}

Notation 3.1. (A) We consider the polynomial ring $S:=K\left[x_{0}, \ldots, x_{r}\right]$ and write $\mathbb{P}_{K}^{r}=$ $\operatorname{Proj}(S)$.

(B) Let $I=I_{\mathcal{C}} \subset S$ denote the homogeneous vanishing ideal of $\mathcal{C} \subset \mathbb{P}_{K}^{r}$ and let $L \subset S$ denote the homogeneous vanishing ideal of the extremal secant line $\mathbb{L}$. Finally, let $J:=L \cap I \subset S$ denote the homogeneous vanishing ideal of $\mathcal{C} \cup \mathbb{L} \subset P_{K}^{r}$.

(C) If $m, n \in \mathbb{Z}$ are integers, we use the convention that $\left(\begin{array}{l}n \\ m\end{array}\right)=0$ for $0<n<m$.

Theorem 3.2. Let $r>3$ and assume that $r+2 \leq d \leq 2 r-3$. For all $i \in\{1, \ldots, r+1\}$ set

$$
a_{i}=(d-r)\left(\begin{array}{c}
r \\
i
\end{array}\right)+\left(\begin{array}{c}
r-1 \\
i-1
\end{array}\right) \text { and } c_{i}=(d-1)\left(\begin{array}{c}
r-1 \\
i
\end{array}\right)-\left(\begin{array}{c}
r-1 \\
i+1
\end{array}\right) .
$$

Then, for all $i \in\{1, \ldots, r\}$ we have

$$
\operatorname{Tor}_{i}^{S}(K, S / I) \simeq K^{u_{i}}(-i-1) \oplus K^{v_{i}}(-i-2) \oplus K^{\left(\begin{array}{r}
r-1 \\
i-1
\end{array}\right)}(-i-d+r-1)
$$

with

and

$$
u_{i} \begin{cases}=\left(\begin{array}{c}
r+1 \\
2
\end{array}\right)-d-1, & \text { for } i=1 \\
=c_{i}-a_{i}, & \text { for } 2 \leq i \leq 2 r-d-1, \\
\leq c_{i}, & \text { for } 2 r-d \leq i \leq r\end{cases}
$$

$$
v_{i}= \begin{cases}0, & \text { for } 1 \leq i \leq 2 r-d-2 \text { and } i=r, \\ u_{i+1}+a_{i+1}-c_{i+1}, & \text { for } 2 r-d-1 \leq i \leq r-2, \\ d-r+1, & \text { for } i=r-1 .\end{cases}
$$

Proof. By our assumption we have $d \leq 2 r-1$. So, by [7, Proposition 3.5] the ring $S / J$ is Cohen-Macaulay. Thus, $[7$, Theorem 4.6] yields that for all $i \in\{1, \ldots, r\}$ we have

$$
\operatorname{Tor}_{i}^{S}(K, S / I) \simeq K^{u_{i}}(-i-1) \oplus K^{v_{i}}(-i-2) \oplus K^{\left(\begin{array}{c}
r-1 \\
i-1
\end{array}\right)}(-i-d+r-1)
$$

with (keep in mind the Notations $3.1(\mathrm{C})$ )

$$
u_{i} \begin{cases}=\left(\begin{array}{c}
r+1 \\
2
\end{array}\right)-d-1, & \text { for } i=1 \\
\leq c_{i}, & \text { for } 2 \leq i \leq r\end{cases}
$$

and

$$
v_{i}= \begin{cases}0, & \text { for } 1 \leq i \leq 2 r-d-2 \text { and } i=r, \\ u_{i+1}+a_{i+1}-c_{i+1}, & \text { for } 2 r-d-1 \leq i \leq r-2, \\ d-r+1, & \text { for } i=r-1 .\end{cases}
$$

(Unfortunately there is a misprint in the formula for $c_{i}$ given in [7, Lemma 4.2] and the formula should be as in the proof of that Lemma.)

It remains to show that $v_{i}=0$ for all $i \leq 2 r-d-2$. So, let $\ell \in S_{1}$ be a generic linear form and set $\mathbb{P}_{K}^{r-1}=\mathbb{H}:=\operatorname{Proj}(S / \ell S)$. Then, according to Corollary 2.6 the scheme

$$
X=\operatorname{Proj}(S /(J+\ell S))=(\mathcal{C} \cup \mathbb{L}) \cap \mathbb{H} \subset \mathbb{H}=\mathbb{P}_{K}^{r-1}
$$

is reduced and consists of

$$
d+1=2(r-1)+1-(2 r-d-2)
$$


points in linearly general position. So, by [13, Theorem 1] the scheme $X \subset \mathbb{P}_{K}^{r-1}=$ $\operatorname{Proj}(S / \ell S)$ satisfies condition $N_{2 r-d-2}$. As $S / J$ is a Cohen-Macaulay ring, $\ell$ is $S / J$-regular and $J S^{\prime} \subset S^{\prime}:=S / \ell S$ is the homogeneous vanishing ideal of $X$. As $X$ satisfies the condition $N_{2 r-d-2}$ it follows (with appropriate integers $d_{1}, \ldots, d_{2 r-d-2}$ ) that

$$
\operatorname{Tor}_{i}^{S^{\prime}}\left(K, S^{\prime} / J S^{\prime}\right) \simeq K^{d_{i}}(-i-1) \text { for all } i \in\{1, \ldots, 2 r-d-2\} .
$$

As $\ell \in S_{1}$ is $S / J$-regular we therefore get

$$
\operatorname{Tor}_{i}^{S}(K, S / J) \simeq K^{d_{i}}(-i-1) \text { for all } i \in\{1, \ldots, 2 r-d-2\} .
$$

According to [7, Proposition 4.1] we have

$$
\operatorname{Tor}_{i}^{S}(K, S / I) \simeq \operatorname{Tor}_{i}^{S}(K, S / J) \oplus K^{\left(\begin{array}{c}
r-1 \\
i-1
\end{array}\right)}(-i-d+r-1)
$$

for all $i \in\{1, \ldots, r\}$. In particular,

$$
\operatorname{Tor}_{i}^{S}(K, S / I) \simeq K^{d_{i}}(-i-1) \oplus K^{\left(\begin{array}{c}
r-1 \\
i-1
\end{array}\right)}(-i-d+r-1)
$$

for all $i \in\{1, \ldots, 2 r-d-2\}$. As $d \geq r+2$ it follows that $v_{i}=0$ for $1 \leq i \leq 2 r-d-2$.

Corollary 3.3. Let $r>3$ and assume that $r+2 \leq d \leq 2 r-2$. Then:

(a) The vanishing ideal $I \subset S$ of $\mathcal{C} \subset \mathbb{P}_{K}^{r}$ is minimally generated by $\left(\begin{array}{c}r+1 \\ 2\end{array}\right)-d-1$ quadrics, at most $(d-1)\left(\begin{array}{l}r \\ 2\end{array}\right)+r-1$ cubics and one form of degree $d-r+2$.

(b) If $d \leq 2 r-3$, there are no cubics in a minimal generating set of the vanishing ideal $I \subset S$.

Proof. (a) : This follows from [7, Proposition 3.5 and Theorem 4.6].

(b) : By Theorem 3.2 we have

$$
\operatorname{Tor}_{1}^{S}(K, S / I) \simeq K^{u_{1}}(-2) \oplus K(-d+r-2)
$$

with $u_{1}=\left(\begin{array}{c}r+1 \\ 2\end{array}\right)-d-1$. This proves our claim.

\section{Surfaces of Degree $\leq 2 r-4$}

Notation 4.1. (A) Let $X \subset \mathbb{P}_{K}^{r}=\operatorname{Proj} S$ with $S=K\left[x_{0}, \ldots, x_{r}\right]$, be an irreducible, reduced non-degenerate projective variety of degree $d>r \geq 4$. By $I$ we denote the homogeneous vanishing ideal of $X$ in $S$ and by $A$ the homogeneous coordinate ring $S / I$ of $X$.

(B) We write $S_{+}$for the irrelevant ideal $\oplus_{n>0} S_{n}=\left(x_{0}, \ldots, x_{n}\right) S$ of $S$. If $M$ is a graded $S$-module and $i \in \mathbb{N}_{0}$, we write $H^{i}(M)$ for the $i$-th local cohomology module of $M$ with respect to $S_{+}$, furnished with its natural grading. If the graded $S$-module $M$ is finitely generated we write $h_{M}^{i}(n)$ for the vector-space dimension $\operatorname{dim}_{K} H^{i}(M)_{n}$ of the $n$-th graded component of $H^{i}(M)$.

Remark 4.2. Keep the above notations and hypothesis. Let $\mathcal{I}_{X} \subset \mathcal{O}_{\mathbb{P}_{K}^{r}}$ be the sheaf of vanishing ideals of $X$. Then, the well known relations between local cohomology and sheaf cohomology yield $h_{A}^{i+1}(n)=h^{i}\left(X, \mathcal{O}_{X}(n)\right)$ for all $i>0$ and all $n \in \mathbb{Z}$ and $h_{A}^{i}(n)=$ $h^{i}\left(\mathbb{P}_{K}^{r}, \mathcal{I}_{X}(n)\right)$ for all $i \neq 0, r$ and all $n \in \mathbb{Z}$.

The following technical result is a generalization of statements shown in $[4,4.4,4.5$, 4.6] for the special case $d=r+1$. 
Proposition 4.3. Let $6 \leq r+1 \leq d \leq 2 r-4$ and let $(f, g) \in S_{1}^{2}$ be a pair of generic linear forms. Then:

(a) $H^{0}(A /(f, g) A)$ is generated by homogeneous elements of degree 2.

(b) $H^{1}(A /(f, g) A)_{n}=0$ for all $n \geq 2$.

(c) For all $(\lambda, \mu) \in K^{2} \backslash\{(0,0)\}$ and all $n \geq 2$ we have

$$
h_{A /(\lambda f+\mu g) A}^{1}(n) \leq \max \left\{h_{A /(\lambda f+\mu g) A}^{1}(n-1)-1,0\right\} .
$$

(d) For all $m \in \mathbb{N}$ and $n \geq h_{A}^{1}(m)+h_{A}^{2}(m-1)+m$ we have

$$
h_{A}^{1}(n) \leq \max \left\{h_{A}^{1}(n-1)-1,0\right\} .
$$

Proof. (a): By our choice of $f$ and $g$ the scheme $Z=\operatorname{Proj}(A /(f, g) A) \subset \mathbb{P}_{K}^{r-2}$ consists of $d=2(r-2)+1-(2 r-d-3)$ points in linearly general position. So, by [13, Theorem 1$]$ this scheme $Z$ satisfies the property $N_{2 r-d-3}$, with $2 r-d-3 \geq 1$. Therefore the vanishing ideal

$$
I_{Z}=(f, g, I)^{\mathrm{sat}} /(f, g) S \subset S /(f, g) S
$$

of $Z$ is generated by homogeneous elements of degree 2 . So, the same is true for

$$
I_{Z} /((f, g, I) /(f, g) S) \simeq(f, g, I)^{\mathrm{sat}} /(f, g, I) \simeq H^{0}(A /(f, g) A) .
$$

(b): The scheme $Z=\operatorname{Proj}(A /(f, g) A) \subset \mathbb{P}_{K}^{r-2}$ consists of $d$ points in semi-uniform position. So, by [6, Lemma $2.4(\mathrm{a})]$ we have:

$$
h_{A /(f, g) A}^{1}(n) \leq \max \{d-1-n(r-2), 0\}
$$

for all $n \geq 0$. As of $d \leq 2 r-4$ we get our claim.

(c): Without loss of generality we may assume that $\mu \neq 0$. We set $\ell:=\lambda f+\mu g$, so that $(f, \ell) A=(f, g) A$. We put $E:=A / \ell A$ and $\bar{E}:=E / H^{0}(E)$. Our first claim is, that $f$ is $\bar{E}$-regular. Indeed, otherwise we would find some $\mathfrak{p} \in \operatorname{Ass} \bar{E}=\operatorname{Ass} E \backslash\left\{S_{+}\right\}$with $f \in \mathfrak{p}$. As $f$ and $\ell$ are $A$-regular, it would follow $\mathfrak{p} \in \operatorname{Ass} A / f A \backslash\left\{S_{+}\right\}$. By $g A \subset(f, \ell) A$ and $f, \ell \in \mathfrak{p}$ we also would have $g \in \mathfrak{p}$ and so $f, g$ would not form a regular sequence with respect to $A_{\mathfrak{p}}$. This would contradict the genericity of the pair $(f, g) \in S_{1}^{2}$.

So, $f$ is $\bar{E}$-regular and we get a short exact sequence

$$
0 \rightarrow \bar{E}(-1) \stackrel{f}{\rightarrow} \bar{E} \rightarrow \bar{E} / f \bar{E} \rightarrow 0 .
$$

In view of the natural isomorphisms $H^{1}(\bar{E}) \simeq H^{1}(E)$ and

$$
H^{1}(\bar{E} / f \bar{E}) \simeq H^{1}(E / f E) \simeq H^{1}(A /(\ell, f) A) \simeq H^{1}(A /(f, g) A)
$$

statement (b) shows that for all $n \geq 2$ there is an exact sequence

$$
0 \rightarrow H^{0}(\bar{E} / f \bar{E})_{n} \rightarrow H^{1}(E)_{n-1} \rightarrow H^{1}(E)_{n} \rightarrow 0 .
$$

Moreover there is an epimorphism of graded $S$-modules $H^{0}(A /(f, g) A) \rightarrow H^{0}(\bar{E} / f \bar{E})$. So, by statement (a) the module $H^{0}(\bar{E} / f \bar{E})$ is generated by homogeneous elements of degree 2. But now, the above sequences and the fact that $H^{1}(E)_{n}=0$ for all $n \gg 0$ imply that for all $n \geq 2$ we have $h_{E}^{1}(n) \leq \max \left\{h_{E}^{1}(n-1)-1,0\right\}$. This is our claim.

(d): Let $(\lambda, \mu) \in K^{2} \backslash\{(0,0)\}$ and set $h=\lambda f+\mu g$. If we apply cohomology to the short exact sequence

$$
0 \rightarrow A(-1) \stackrel{h}{\rightarrow} A \rightarrow A / h A \rightarrow A
$$


we get exact sequences

$$
H^{1}(A)_{n-1} \stackrel{h}{\rightarrow} H^{1}(A)_{n} \rightarrow H^{1}(A / h A) \rightarrow H^{2}(A)_{n-1} .
$$

Applying this with $n=m$, we see that

$$
h_{A / h A}^{1}(m) \leq h_{A}^{1}(m)+h_{A}^{2}(m-1) .
$$

So, by statement (c) we get $h_{A / h A}^{1}(n)=0$ and hence an epimorphism $H^{1}(A)_{n-1} \rightarrow H^{1}(A)_{n}$ for all $n \geq h_{A}^{1}(m)+h_{A}^{2}(m-1)+m$ and all pairs $(\lambda, \mu) \in K^{2} \backslash\{(0,0)\}$. By [4, Lemma $3.2]$ we may conclude that for all $n \geq h_{A}^{1}(m)+h_{A}^{2}(m-1)+m$ we have

$$
h_{A}^{1}(n) \leq \max \left\{h_{A}^{1}(n-1)-1,0\right\} .
$$

Notation and Remark 4.4. (A) Later we shall be interested in the place, at which the Hartshorne-Rao function $n \mapsto h_{A}^{1}(n)=h^{1}\left(\mathbb{P}_{K}^{r}, \mathcal{I}_{X}(n)\right)$ definitively descents, that is in the invariant

$$
\delta(X):=\inf \left\{m \in \mathbb{Z} \mid h_{A}^{1}(n) \leq \max \left\{h_{A}^{1}(n-1)-1,0\right\} \text { for all } n>m\right\} .
$$

(B) If $T=\oplus_{n \in \mathbb{Z}} T_{n}$ is a graded $S$-module, we define the beginning and the end of $T$ by

$$
\operatorname{beg}(T):=\inf \left\{n \in \mathbb{Z} \mid T_{n} \neq 0\right\}, \operatorname{end}(T)=\sup \left\{n \in \mathbb{Z} \mid T_{n} \neq 0\right\} .
$$

Lemma 4.5. Let $\mathbb{H}:=\mathbb{P}_{K}^{r-1} \subset \mathbb{P}_{K}^{r}$ be a hyperplane such that the intersection curve $\mathcal{C}:=X \cap \mathbb{H} \subset \mathbb{H}$ is reduced and irreducible. Then:

(a) $\operatorname{reg} \mathcal{C} \leq \min \{\operatorname{reg} X, d-r+3\}$.

(b) $\delta(X) \leq \min \left\{\operatorname{reg} \mathcal{C}-1\right.$, end $\left.H^{1}(A)\right\} \leq \operatorname{reg} X-2$.

Proof. (a): The relation $\operatorname{reg} \mathcal{C} \leq \operatorname{reg} X$ is well known. Moreover, by [15] we have

$$
\operatorname{reg} \mathcal{C} \leq \operatorname{deg} \mathcal{C}-(r-1)+2=d-r+3 .
$$

(b): By Mumford's Lemma on the descent of the Hartshorne-Rao function

$$
n \mapsto h_{A}^{1}(n)=h^{1}\left(\mathbb{P}_{K}^{r}, \mathcal{I}_{X}(n)\right)
$$

(cf. [19, page 102, statement \#']) we have

$$
h_{A}^{1}(n) \leq \max \left\{h_{A}^{1}(n-1)-1,0\right\} \text { for all } n>\operatorname{reg} \mathcal{C}-1
$$

so that $\delta(X) \leq \operatorname{reg} \mathcal{C}-1$. Clearly $\delta(X) \leq$ end $H^{1}(A) \leq \operatorname{reg} X-2$.

Corollary 4.6. Let $4<d<2 r-4$. Then $\delta(X) \leq \min \left\{d-r+2, h_{A}^{1}(1)+h_{A}^{2}(0)\right\}$.

Proof. Apply statements (a) and (b) of Lemma 4.5 and Proposition 4.3 (d) with $m=$ 1.

We close this section with another result which helps to pave the way for our investigations of surfaces of maximal sectional regularity. 
Notation 4.7. (A) We introduce the invariant

$$
e(X):=\sum_{x \in X, x \text { closed }} \lambda_{\mathcal{O}_{X, x}}\left(H_{\mathfrak{m}_{X, x}}^{1}\left(\mathcal{O}_{X, x}\right)\right)(<\infty)
$$

which counts the number of non-Cohen-Macaulay points on $X$ in a weighted way.

(B) By $\sigma(X)$ we denote the sectional genus of $X$, that is the arithmetic genus of the generic hyperplane section of $X$. So, we have that

$$
\sigma(X)=h_{A / f A}^{2}(0),
$$

for a generic linear form $f \in S_{1}$.

(C) We denote the normal locus, the Cohen-Macaulay locus and the singular locus of $X$ respectively by $\operatorname{Nor}(X), \operatorname{CM}(X)$ and $\operatorname{Sing}(X)$.

Remark 4.8. (A) According to [3, Proposition 5.9] we have

$$
e(X) \leq h_{A}^{2}(n-1) \leq \max \left\{e(X), h_{A}^{2}(n)-1\right\} \text { for all } n \leq 0 .
$$

(B) As $X$ is a surface, we have $\#(X \backslash \operatorname{Nor}(X))<\infty$ if and only if $\# \operatorname{Sing}(X)<\infty$.

(C) Moreover, by Bertini's Theorem the generic hyperplane section $\mathcal{C}=X \cap \mathbb{P}_{K}^{r-1}$ is smooth, if and only if $\operatorname{Sing}(X)$ is a finite set.

(D) For a generic linear form $f \in S_{1}$ we have $h_{A / f A}^{1}(0)=0$ and $h_{A / f A}^{3}(0)=0$. As $\sigma(X)=h_{A / f A}^{2}(0)$, the short exact sequence $0 \rightarrow A(-1) \rightarrow A \rightarrow A / f A \rightarrow 0$ yields:

$$
\sigma(X)=h_{A}^{2}(0)-h_{A}^{2}(-1)-\left(h_{A}^{3}(0)-h_{A}^{3}(-1)\right) .
$$

In particular, $\sigma(X)$ is the sectional genus of the polarized pair $\left(X, \mathcal{O}_{X}(1)\right)$ in the sense of Fujita [11].

Proposition 4.9. Assume that $\sigma(X)=0$. Let $f \in S_{1}$ be a generic linear form and set $\mathcal{C}=\operatorname{Proj} A / f A$. Then:

(a) (i) $h_{A}^{2}(-1)=h_{A}^{2}(0)$,

(ii) $h_{A}^{2}(0)-h_{A}^{2}(1)=h_{A / f A}^{1}(1)-h_{A}^{1}(1) \geq 0$,

(iii) $h_{A}^{2}(n) \leq \max \left\{0, h_{A}^{2}(n-1)-1\right\}$ for all $n \geq 2$,

(iv) $h_{A}^{3}(n)=0$ for all $n \geq-1$.

(b) $\mathcal{C} \simeq \mathbb{P}_{K}^{1}$ if and only if $\# \operatorname{Sing}(X)<\infty$.

(c) If $4<r<d \leq 2 r-2$ and \# $\operatorname{Sing}(X)<\infty$, then $h_{A}^{2}(n)=e(X)$ for all $n \leq 0$.

Proof. (a): We have $h_{A / f A}^{2}(0)=\sigma(X)=0$, so that $h_{A / f A}^{2}(n)=0$ for all $n \geq 0$. As $h_{A / f A}^{1}(n)=0$ for all $n \leq 0$ and $h_{A}^{3}(n)=0$ for all $n \gg 0$ the statements (i), (ii), and (iv) follow immediately from the short exact sequence $0 \rightarrow A(-1) \rightarrow A \rightarrow A / f A \rightarrow 0$. Statement (iii) follows from [5, Proposition 3.5 (b)].

(b): As $\mathcal{C}$ is of arithmetic genus 0 , it is smooth if and only if it is isomorphic to $\mathbb{P}_{K}^{1}$. Now, we conclude by Remark 4.8 (b).

(c): According to [5, Proposition $3.8(\mathrm{~b})]$ we have $h_{A}^{2}(n)=e(X)$ for all $n<0$. We thus get our claim by statement (a) (i). 


\section{Surfaces of maximal Sectional Regularity}

We keep the notations and hypothesis of the previous section.

Definition 5.1. We define the sectional regularity $\operatorname{sreg} X$ as the least value of reg $X \cap \mathbb{H}$, where $\mathbb{H}=\mathbb{P}_{K}^{r-1} \subset \mathbb{P}_{K}^{r}$ runs through all hyperplanes of $\mathbb{P}_{K}^{r}$. Thus we may write

$$
\operatorname{sreg} X=\min \left\{\operatorname{reg}(\operatorname{Proj}(A / f A)) \mid f \in S_{1} \backslash\{0\}\right\} .
$$

Remark 5.2. (A) For all $f \in S_{1} \backslash\{0\}$ we have

$$
\operatorname{reg}(\operatorname{Proj}(A / f A))=\max \left\{\text { end } H^{1}(A / f A)+2, \text { end } H^{2}(A / f A)+3\right\}
$$

and so the short exact sequences

$$
\begin{aligned}
H^{1}(A)_{n-1} & \stackrel{f}{\rightarrow} H^{1}(A)_{n} \rightarrow H^{1}(A / f A)_{n} \rightarrow H^{2}(A)_{n-1} \stackrel{f}{\rightarrow} H^{2}(A)_{n} \\
& \rightarrow H^{2}(A / f A)_{n} \rightarrow H^{3}(A)_{n-1} \stackrel{f}{\rightarrow} H^{3}(A)_{n}
\end{aligned}
$$

yield that the set

$$
\left\{f \in S_{1} \backslash\{0\} \mid \operatorname{reg}(\operatorname{Proj}(A / f A))=\operatorname{sreg} X\right\}
$$

is dense and open in $S_{1}$. In particular, for a generic hyperplane section $\mathcal{C}=X \cap \mathbb{P}_{K}^{r-1}$ of $X$ we have $\operatorname{reg} \mathcal{C}=\operatorname{sreg} X$. So that $\operatorname{sreg} X$ is the regularity of the generic hyperplane section of $X$.

(B) As the generic hyperplane section $\mathcal{C}=X \cap \mathbb{P}_{K}^{r-1}$ of $X$ is reduced and irreducible it follows

$$
\begin{aligned}
\operatorname{sreg} X \leq & \operatorname{reg} X, \text { with equality if } X \text { is of arithmetic depth }>1 ; \\
& \operatorname{sreg} X \leq d-r+3, \text { and } \delta(X) \leq \operatorname{sreg} X-1
\end{aligned}
$$

Definition 5.3. In view of Remark $5.2(\mathrm{~B})$ it makes sense to say that the surface $X \subset \mathbb{P}_{K}^{r}$ is of maximal sectional regularity if sreg $X=d-r+3$. It is equivalent to say that the generic hyperplane section $\mathcal{C}=X \cap \mathbb{P}_{K}^{r-1} \subset \mathbb{P}_{K}^{r-1}$ satisfies reg $\mathcal{C}=d-r+3$ and thus is a curve of maximal regularity.

Remark 5.4. (A) Assume that the surface $X \subset \mathbb{P}_{K}^{r}$ is of maximal sectional regularity. Assume that $d=\operatorname{deg} X>r$. Then, the generic hyperplane section curve $\mathcal{C}=X \cap \mathbb{P}_{K}^{r-1} \subset$ $\mathbb{P}_{K}^{r-1}$ is of maximal regularity and of degree $d>r-1$. So by [15, Table on p. 505] the curve $\mathcal{C}$ is smooth and rational. Moreover, it follows (cf. Proposition 4.9 (b))

$$
\mathcal{C} \simeq \mathbb{P}_{K}^{1}, \sigma(X)=0, \text { and } \# \operatorname{Sing}(X)<\infty
$$

(B) If a surface $X \subset \mathbb{P}_{K}^{r}$ of degree $d$ is of maximal sectional regularity, it satisfies reg $X \geq$ $d-r+3$ by Remark $5.2(\mathrm{~B})$. On the other hand there are examples showing that reg $X=$ $d-r+3$ need not imply that $X$ is of maximal sectional regularity (cf. Example $7.1(\mathrm{~A})$ ). So, being of maximal sectional regularity is strictly stronger than having the maximal regularity conjectured by Eisenbud and Gôto.

Let $X \subset \mathbb{P}_{K}^{r}$ be as above. According to Bertin [1] a line $\mathbb{L}=\mathbb{P}_{K}^{1} \subset \mathbb{P}_{K}^{r}$ is called an extremal secant line to $X$ if $\lambda\left(\mathcal{O}_{X \cap \mathbb{L}}\right)=d-r+3$. Concerning the relation between the condition that $X$ is of maximal sectional regularity and that $X$ has an extremal secant line we have the following result. 
Proposition 5.5. Let $4<r<d$, let $\mathbb{L} \subset \mathbb{P}_{K}^{r}$ be a line and let $\mathcal{H}$ be the linear system of all hyperplanes $\mathbb{H} \subset \mathbb{P}_{K}^{r}$ with $\mathbb{L} \subset \mathbb{H}$. Consider the following four statements:

(i) For a generic $\mathbb{H} \in \mathcal{H}$ the curve $\mathcal{C}=X \cap \mathbb{H} \subset \mathbb{H}$ is reduced, irreducible, of regularity $d-r+3$ and $\mathbb{L}$ is an extremal secant line to $\mathcal{C}$.

(ii) There is some $\mathbb{H} \in \mathcal{H}$ such that the curve $\mathcal{C}=X \cap \mathbb{H} \subset \mathbb{H}$ satisfies the requirements of statement (i).

(iii) $\mathbb{L}$ is a secant line to $X$ with $\mathbb{L} \cap X \subseteq \mathrm{CM}(X)$.

(iv) $\mathbb{L} \nsubseteq X, \mathbb{L} \cap X \subseteq \mathrm{CM}(X)$ and $\lambda\left(\mathcal{O}_{X \cap \mathbb{L}}\right) \geq d-r+3$.

Then

(a) We have the implications (i) $\Rightarrow$ (ii) $\Rightarrow$ (iii) $\Rightarrow$ (iv).

(b) If Char $K=0$, then all of the four statements are equivalent.

Proof. (a): It suffices to show that the implication "(ii) $\Rightarrow$ (iii)" holds. So, let $\mathbb{H} \in \mathcal{H}$ be as in statement (ii). Then $\mathcal{C}=X \cap \mathbb{H} \subset \mathbb{H}$ is a reduced and irreducible curve with $\operatorname{deg} \mathcal{C}=d$ and $\operatorname{reg} \mathcal{C}=\lambda\left(\mathcal{O}_{X \cap \mathbb{L}}\right)=d-r+3$. As $X$ is a surface and $\mathcal{C}$ is Cohen-Macaulay and locally cut out by one equation from $X$, we must have $X \cap \mathbb{H}=\mathcal{C} \subseteq \operatorname{CM}(X)$, whence $X \cap \mathbb{L} \subseteq \mathrm{CM}(X)$. Moreover

$$
\lambda\left(\mathcal{O}_{X \cap \mathbb{L}}\right)=\lambda\left(\mathcal{O}_{(X \cap \mathbb{H}) \cap \mathbb{L}}\right)=\lambda\left(\mathcal{O}_{\mathcal{C} \cap \mathbb{L}}\right)=d-r+3 .
$$

(b): It suffices to prove the implication "(iv) $\Rightarrow$ (i)". So let $\mathbb{L}$ be as in statement (iv). As $\#(\mathbb{L} \cap X)<\infty$ and Char $K=0$ it follows by Bertini Theorems that the curve $\mathcal{C}=X \cap \mathbb{H} \subset \mathbb{H}$ is reduced outside $\mathbb{L} \cap X$ and irreducible for generic $\mathbb{H} \in \mathcal{H}$. As $\mathbb{L} \cap X \subset \mathrm{CM}(X)$ and $\mathcal{C}$ is locally cut out from $X$ by one equation, it follows that $\mathcal{C}$ is also reduced in all points $x \in \mathbb{L} \cap X$. So $\mathcal{C}$ is reduced and irreducible at all. Moreover $\operatorname{reg} \mathcal{C} \geq \lambda\left(\mathcal{O}_{\mathcal{C} \cap \mathbb{L}}\right)=\lambda\left(\mathcal{O}_{X \cap \mathbb{L}}\right) \geq d-r+3=\operatorname{deg} \mathcal{C}-(r-1)+2 \geq \operatorname{reg} \mathcal{C}($ cf. [15]). This proves our claim.

Corollary 5.6. Let $4<r<d$. If there is a hyperplane $\mathbb{H} \subset \mathbb{P}_{K}^{r}$ such that the curve $\mathcal{C}=X \cap \mathbb{H} \subset \mathbb{H}$ is reduced and irreducible and of regularity $d-r+3$, then $X$ admits an extremal secant line $\mathbb{L} \subset \mathbb{P}_{K}^{r}$ such that $X \cap \mathbb{L} \subseteq \mathrm{CM}(X)$. If Char $K=0$, the converse is true also.

Proof. Clear from Proposition 5.5 and the fact that a curve of maximal regularity of degree $d$ in $\mathbb{P}_{K}^{r-1}$ has an extremal secant line (cf. [15]).

Corollary 5.7. Let $4<r<d$. If $X$ is of maximal sectional regularity, it admits an extremal secant line $\mathbb{L}$ such that $X \cap \mathbb{L} \subseteq \mathrm{CM}(X)$.

Proof. Clear from Corollary 5.6.

So, in this section, we are actually interested in surfaces $X$ having not only one extremal secant line, but "many of them". Moreover we wish not to restrict the type of singularities of $X$ nor the characteristic of the base field $K$. We thus cannot make use of the classification given in [1] and [2].

The technical key result of the present section is Proposition 5.9 below. The natural aim to prove statement (a) of this Proposition would be to apply the Socle Lemma of Huneke and Ulrich (cf. [17, Corollary (3.11) (i)]). But this would mean that we had to assume that the base field $K$ is of characteristic 0 . As we prefer a characteristic free 
approach, we shall attack the proof of Proposition 5.9 in a slightly different way. We namely first prove the following lemma, which follows easily from a result of Kreuzer (cf. [18]).

For a graded $S$-module $M$ let

$$
\operatorname{soc} M:=0:_{M} S_{+}=\operatorname{Hom}_{S}(K, M)
$$

denote the socle of $M$.

Lemma 5.8. Let $\ell \in S_{1}$ be a generic linear form and let $T$ be a finitely generated graded $S$-module. Then for each integer $n<\operatorname{beg} \operatorname{soc}(T / \ell T)$ we have $\left(0:_{T} \ell\right)_{n} \subset \ell T_{n-1}$.

Proof. Let $n<\operatorname{beg} \operatorname{soc}(T / \ell T)$ and $t \in\left(0:_{T} \ell\right)_{n}$. Then $\ell t=0$. As $\ell \in S_{1}$ is generic and $K$ is infinite, we may apply [18, Corollary $(1.2)(\mathrm{b})]$ to the finitely generated graded $S$-module $T$ and get that $\ell^{\prime} t \in \ell T=0$ for all $\ell^{\prime} \in S_{1}$. Therefore $t+\ell T \in(\operatorname{soc}(T / \ell T))_{n}=0$, thus $t \in \ell T$.

Proposition 5.9. Assume that $4<r<d$ and the surface $X$ is of maximal sectional regularity. Let $\ell \in S_{1}$ be a generic linear form and set $U:=\left(0: H_{H^{1}(A)} \ell\right)(-1)$. Then:

(a) If $d \leq 2 r-4$, then $U$ is minimally generated by at most $(d-1)\left(\begin{array}{c}r-1 \\ 2\end{array}\right)+r-2$ forms of degree 3 and at most one form of degree $d-r+3$.

(b) If $d \leq 2 r-5$, then $U$ is generated by at most one single form of degree $d-r+3$.

Proof. Let $\mathbb{H}:=\operatorname{Proj} S / \ell S=\mathbb{P}_{K}^{r-1}$, so that

$$
\mathcal{C}:=X \cap \mathbb{H}=\operatorname{Proj} A / \ell A \subset \mathbb{H}=\mathbb{P}_{K}^{r-1}
$$

is a curve of maximal regularity and degree $d$ in $\mathbb{P}_{K}^{r-1}$. If we apply cohomology to the short exact sequence

$$
0 \rightarrow A(-1) \stackrel{\ell}{\longrightarrow} A \rightarrow A / \ell A \rightarrow 0
$$

we get an isomorphism of graded $S$-modules $H^{0}(A / \ell A) \simeq U$. Now, let

$$
I_{\mathcal{C}}:=(I+\ell S)^{\mathrm{sat}} / \ell S \subset S / \ell S
$$

be the homogeneous vanishing ideal of $\mathcal{C}$. Then the isomorphisms

$$
I_{\mathcal{C}} /((I+\ell S) / \ell S) \simeq(I+\ell S)^{\mathrm{sat}} /(I+\ell S)=H^{0}(S /(I+\ell S)) \simeq H^{0}(A / \ell A)
$$

show that there is an epimorphism of graded $S$-modules $I_{\mathcal{C}} \rightarrow U$. As

$$
\operatorname{deg} \mathcal{C}=d \leq 2 r-4=2(r-1)-2
$$

we get from Corollary 3.3 that $I_{\mathcal{C}} \subset S / \ell S$ is minimally generated by $\left(\begin{array}{l}r \\ 2\end{array}\right)-d-1$ quadrics, at most $(d-1)\left(\begin{array}{c}r-1 \\ 2\end{array}\right)+r-2$ cubics and one form of degree $d-r+3$. Moreover the cubics are not needed if $\operatorname{deg} \mathcal{C}=d \leq 2(r-1)-3=2 r-5$. In view of the previous epimorphism $I_{\mathcal{C}} \rightarrow U$ it therefore remains to show that beg $U \geq 3$ or - equivalently - that $\operatorname{beg}\left(0:_{H^{1}(A)} \ell\right) \geq 2$. Observe that $H^{1}(A / \ell A) \simeq H^{1}\left(A_{\mathcal{C}}\right)$, where $A_{\mathcal{C}}=(S / \ell S) / I_{\mathcal{C}} \simeq(A / \ell A) / H^{0}(A / \ell A)$ is the homogeneous coordinate ring of $\mathcal{C} \subset \mathbb{P}_{K}^{r-1}$. Now, applying [7, Proposition 3.5 and Theorem 3.3] to $\mathcal{C}$, we get $\operatorname{soc} H^{1}\left(A_{\mathcal{C}}\right) \simeq K(r-d-1)$ and hence $\operatorname{soc} H^{1}(A / \ell A) \simeq K(r-d-1)$. Thus soc $H^{1}(A) / \ell H^{1}(A) \simeq K(r-d-1)$, so that $\operatorname{beg}\left(\operatorname{soc} H^{1}(A) / \ell H^{1}(A)\right)=d-r+1 \geq 2$.

Observe that beg $H^{1}(A) \geq 1$. Assume now, that beg $U \leq 2$. Then, there is an element $u \in U_{2} \backslash\{0\}=\left(0::_{H^{1}(A)} \ell\right)_{1} \backslash\{0\}=H^{1}(A)_{1} \backslash\{0\}$. According to Lemma 5.8 we get $u \in \ell H^{1}(A)_{0}=\ell \cdot 0=0$, a contradiction. 
In the next result we use the invariant $\delta(X)$ introduced in Notation 4.4.

Theorem 5.10. Assume that $4<r<d$ and the surface $X \subset \mathbb{P}_{K}^{r}$ is of maximal sectional regularity, of arithmetic depth one and satisfies $\delta(X) \leq d-r+1$. Then $d>2 r-5$.

Proof. Assume that all the hypotheses are satisfied and that $d \leq 2 r-5$. Let $\ell \in S_{1}$ and $U$ be as in Proposition 5.9. Then statement (b) of this Proposition yields that $U$ is generated by a form of degree $d-r+3$, or vanishes. We consider the exact sequences

$$
0 \rightarrow U_{n} \rightarrow H^{1}(A)_{n-1} \stackrel{\ell}{\rightarrow} H^{1}(A)_{n}
$$

for all $n \in \mathbb{Z}$.

As $U_{n}=0$ for all $n \leq d-r+2$ we have $h_{A}^{1}(n) \geq h_{A}^{1}(n-1)$ for all of these $n$. As $\delta(X) \leq d-r+1$ we also have $h_{A}^{1}(n) \leq \max \left\{h_{A}^{1}(n-1)-1,0\right\}$ for all $n \geq d-r+2$. Both statements together yield $H^{1}(A)=0$, and this contradicts the hypothesis that $X$ is of arithmetic depth one.

Corollary 5.11. Let $4<r<d$ and assume that the surface $X \subset \mathbb{P}_{K}^{r}$ is of maximal sectional regularity and of arithmetic depth one. Assume that $h_{A}^{1}(1)+h_{A}^{2}(0) \leq d-r+1$ or end $\left(H^{1}(A)\right) \leq d-r+2$. Then $d>2 r-5$.

Proof. This is immediate by Lemma 4.5 (b), Proposition 4.6 and Theorem 5.10.

Reminder 5.12. (A) Let $h \in \mathbb{N}_{0}$. A non-degenerate irreducible projective surface $Y \subset$ $\mathbb{P}_{K}^{r+h}$ is called a projecting surface for $X$, if there is a linear projection $\pi: \mathbb{P}_{K}^{r+h} \backslash \mathbb{P}_{K}^{h-1} \rightarrow \mathbb{P}_{K}^{r}$ whose center $\mathbb{P}_{K}^{h-1}$ is disjoint to $Y$ and such that by restricting $\pi$ we get an isomorphism $\pi \uparrow$ : $Y \stackrel{\cong}{\rightarrow} X$. Such projecting surfaces $Y \subset \mathbb{P}_{K}^{r+h}$ for $X$ exist if and only if $h \leq h^{1}\left(\mathbb{P}_{K}^{r}, \mathcal{I}_{X}(1)\right)=$ $h_{A}^{1}(1)$. If $h=h_{A}^{1}(1)$, we speak of maximal projecting surfaces for $X$. Moreover, we call $h_{A}^{1}(1)$ the linear deficiency of $X$.

(B) Now, let $h=h_{A}^{1}(1)$ and let $Y \subset \mathbb{P}_{K}^{r+h}$ be a maximal projecting surface for $Y$. If $B$ denotes the homogeneous coordinate ring for $Y$, we have the inclusions

$$
A \hookrightarrow B=K\left[D(A)_{1}\right] \subseteq D(A)=\oplus_{n \in \mathbb{Z}} H^{0}\left(X, \mathcal{O}_{X}(n)\right),
$$

where $D(A):=\lim _{\longrightarrow} \operatorname{Hom}_{S}\left(\left(S_{+}\right)^{n}, A\right)$ denotes the $S_{+}$-transform of $A$. Observe that

$$
\operatorname{dim}_{K} D(A)_{1}=r+h+1 \text { and that } \operatorname{deg} Y=\operatorname{deg} X=d .
$$

As $Y \subset \mathbb{P}_{K}^{r+h}$ is non-degenerate we have $d-\operatorname{codim} Y \geq 1$, whence $d \geq r+h_{A}^{1}(1)-1$.

Corollary 5.13. Let $4<r<d$ and assume that the surface $X \subset \mathbb{P}_{K}^{r}$ is of maximal sectional regularity, of arithmetic depth one and Cohen-Macaulay. Then $d>2 r-5$.

Proof. By Reminder 5.12 (B) we have $h_{A}^{1}(1) \leq d-r+1$. By Remark 5.4 (A) we have $\sigma(X)=0$ and \# $\operatorname{Sing}(X)<\infty$. So, by Proposition 4.9 (c) we have $h_{A}^{2}(0)=e(X)$. As $X$ is Cohen-Macaulay, we have $e(X)=0$. Now, we conclude by Corollary 5.11

\section{SurfaCES With HIGH LINEAR DEFICIENCY}

We keep all notation and hypotheses of sections 4 and 5. According to Reminder 5.12 (B) we have $h_{A}^{1}(1) \leq d-r+1$. In this section, we shall consider the situation in which $h_{A}^{1}(1)$ is "close to being maximal", more precisely the cases in which

$$
h_{A}^{1}(1) \in\{d-r-1, d-r, d-r+1\} .
$$


We start by recalling a few facts on certain surfaces $Y \subset \mathbb{P}_{K}^{s}$ of degree $s+1$.

Reminder 6.1. (A) Let $s \geq 5$ and let $Y \subset \mathbb{P}_{K}^{s}$ be a non-degenerate and irreducible surface of degree $s+1$ with homogeneous coordinate ring $B$. Assume that $Y$ is a maximal projecting surface of the surface $X \subset \mathbb{P}_{K}^{r}$ so that $r \leq s$ and $h_{B}^{1}(1)=0$. Then, according to [4] the surface $Y$ must be one of the six types I, IIA, IIA', IIIA, IVA0, IVA1 which were introduced there. For these 6 types one has the following facts (cf. [4, Propositions $4.2,4.11,5.5$ and 5.6]):

(i) Type I: $Y$ is arithmetically Cohen-Macaulay, sreg $Y=3$ and $\sigma(Y)=2$.

(ii) Type IIA: $Y$ is Cohen-Macaulay, depth $B=2, \operatorname{sreg} Y=3, h_{B}^{2}(0)=1, h_{B}^{2}(n)=0$ for all $n \neq 0$ and $\sigma(Y)=2$.

(iii) Type IIA': $\operatorname{sreg} Y=3$, depth $B=2, h_{B}^{2}(n)=e(Y)=1$ for all $n \leq 0, h_{B}^{2}(n)=0$ for all $n>0$ and $\sigma(Y)=0$.

(iv) Type IIIA: $\operatorname{sreg} Y=3$, depth $B=2, h_{B}^{2}(n)=e(Y)=2$ for all $n \leq 0, h_{B}^{2}(n)=0$ for all $n>0$ and $\sigma(Y)=0$.

(v) Type IVA1: $\operatorname{sreg} Y=4, \operatorname{depth} B=2, h_{B}^{2}(n)=e(Y)=3$ for all $n \leq 0, h_{B}^{2}(1)=$ $1, h_{B}^{2}(n)=0$ for all $n>1$ and $\sigma(Y)=0$.

(vi) Type IVA0: $\operatorname{sreg} Y=4$, depth $B=1, h_{B}^{1}(1)=1, h_{B}^{1}(n)=0$ for all $n \neq 0$ and the values of $h_{B}^{2}(n), e(Y)$ and $\sigma(Y)$ are as in statement (iv).

(B) Again, let $s \geq 5$ and $Y \subset \mathbb{P}_{K}^{s}$ a non-degenerate reduced and irreducible surface of degree $s+1$ with homogeneous coordinate ring $B$. Assume this time that $h_{B}^{1}(n)=2$ for $n=1,2$ and $h_{B}^{1}(n)=0$ for $n \neq 1,2$. Then, according to [4], the surface $Y$ must be one of the types IIC or IVC which were introduced there. For these two types one has (cf. [4, Propositions 5.5, 5.6]).

(i) Type IIIC: $Y$ is Cohen-Macaulay, $\operatorname{sreg} Y=3, H^{2}(B)=0$ and $\sigma(Y)=0$.

(ii) Type IVC: $Y$ is Cohen-Macaulay, $\operatorname{sreg} Y=4, H^{2}(B)=0$ and $\sigma(Y)=0$.

In both cases we also have $h_{B}^{3}(n)=0$ for all $n \geq-1$. By our assumption end $H^{1}(B)=2$, and so in both cases $\operatorname{reg} Y=4$.

Lemma 6.2. Let $A:=A$. Let $h \in\left\{0, \ldots, h_{A}^{1}(1)\right\}$ and let $Y \subset \mathbb{P}_{K}^{r+h}$ be a projecting surface for $X$. Let $B$ denote the homogeneous coordinate ring of $Y$. Then:

(a) $h_{B}^{1}(1)=h_{A}^{1}(1)-h$.

(b) $h_{B}^{i}(n)=h_{A}^{i}(n)$ for all $i \geq 2$ and all $n \in \mathbb{Z}$.

(c) $\sigma(Y)=\sigma(X)$.

(d) $e(Y)=e(X)$.

(e) $\operatorname{sreg} Y \leq \operatorname{sreg} X \leq d-r+3$.

Proof. (a): We have the inclusions $A \hookrightarrow B \hookrightarrow D(A)=D(B)$, in which $D(\cdot)$ denotes the formation of $S_{+}$-transform. It follows

$$
h_{B}^{1}(1)=\operatorname{dim}_{K} D(B)_{1}-\operatorname{dim}_{K} B_{1}=\operatorname{dim}_{K} D(A)_{1}-\left(\operatorname{dim}_{K} A_{1}+h\right)=h_{A}^{1}(1)-h .
$$

(b): As $D(A)=D(B)$ we have $H^{i}(B)=H^{i}(A)$ for all $i>1$.

(c): Clear from statement (b) and Remark 6.1.

(d): Follows from $Y \simeq X$.

(e): In view of Remark 5.2 (B) it is enough to show the first inequality. We write $C:=B / A$, choose $f \in S_{1} \backslash\{0\}$ and consider the $S_{+}$-torsion modules $V:=C / f C$ and 
$U:=\left(0:_{C} f\right)(-1)$. As the multiplication maps $f: A(-1) \rightarrow A$ and $f: B(-1) \rightarrow B$ are injective the snake lemma yields a short exact sequence of graded $S$-modules

$$
0 \rightarrow U \rightarrow A / f A \rightarrow B / f B \rightarrow V \rightarrow 0 .
$$

As $U$ and $V$ are $S_{+}$-torsion we thus get an epimorphism $H^{1}(A / f A) \rightarrow H^{1}(B / f B)$ and an isomorphism $H^{2}(A / f A) \simeq H^{2}(B / f B)$. So end $H^{i}(A / f A) \geq$ end $H^{i}(B / f B), i=1,2$. Whence $\operatorname{reg} \operatorname{Proj}(A / f A) \geq \operatorname{reg} \operatorname{Proj}(B / f B) \geq \operatorname{sreg} Y$, as required.

Theorem 6.3. Let $4<r<d$, set $h:=h_{A}^{1}(1)$ and let $Y \subset \mathbb{P}_{K}^{r+h}$ be a maximal projecting surface for $X$.

(a) If $h=d-r+1$, then $Y \subset \mathbb{P}_{K}^{d+1}$ is a smooth rational normal surface scroll, hence $X$ is smooth and rational with $H^{2}(A)=0$ and $\sigma(X)=0$.

(b) If $h=d-r$, then either

(i) $Y \subset \mathbb{P}_{K}^{d}$ is a non-normal Del Pezzo surface in the sense of [9], in particular $X$ is Cohen-Macaulay, $X \backslash \operatorname{Nor}(X)$ is a line, $H^{2}(A)=0$ and $\sigma(X)=1$, or

(ii) $Y \subset \mathbb{P}_{K}^{d}$ is a surface of almost minimal degree of arithmetic depth 2 , in particular $\mathrm{CM}(X)=\operatorname{Nor}(X), X \backslash \operatorname{Nor}(X)$ consists of a single point, $h_{A}^{2}(n)=$ $e(X)=1$ for all $n \leq 0, h_{A}^{2}(n)=0$ for all $n>0$, and $\sigma(X)=0$.

(c) If $h=d-r-1$, then $Y \subset \mathbb{P}_{K}^{d-1}$ is one of the six types (i) - (vi) listed in Reminder 6.1 (A) and:

(i) If $Y$ is of type I, then $X$ is Cohen-Macaulay, $H^{2}(A)=0$ and $\sigma(X)=2$.

(ii) If $Y$ is of type II $A, X$ is Cohen-Macaulay, $h_{A}^{2}(0)=1, h_{A}^{2}(n)=0$ for all $n \neq 0$ and $\sigma(X)=1$.

(iii) If $Y$ is of type II $A^{\prime}, h_{A}^{2}(n)=e(X)=1$ for all $n \leq 0, h_{A}^{2}(n)=0$ for all $n>0$ and $\sigma(X)=1$.

(iv) If $Y$ is of type III $A$ or IV $A 0, h_{A}^{2}(n)=e(X)=2$ for all $n \leq 0, h_{A}^{2}(n)=0$ for all $n>0$ and $\sigma(X)=0$.

(v) If $Y$ is of type IV A1, $h_{A}^{2}(n)=e(X)=3$ for all $n \leq 0, h_{A}^{2}(1)=1, h_{A}^{2}(n)=0$ for all $n>1$ and $\sigma(X)=0$.

Proof. Let $B$ be the homogeneous coordinate ring of $Y$. According to Lemma 6.2 (a) we have $h_{B}^{1}(1)=0$.

(a): Let $h=d-r+1$. Then, $Y \subset \mathbb{P}_{K}^{d+1}$ is of degree $d$ and hence a surface of minimal degree. As $d>5, Y$ cannot be the Veronese surface and hence, by the classification of varieties of minimal degree, is either a smooth scroll or a cone over a rational normal curve (cf. [16, Theorem 19.9]) . In the latter case, each line in $\mathbb{P}_{K}^{d+1}$ passing through the vertex of $Y$ would be tangent to $Y$ and thus no linear projection $\mathbb{P}_{K}^{d+1} \backslash \mathbb{P}^{d-r} \rightarrow \mathbb{P}_{K}^{r}$ with $\mathbb{P}_{K}^{d-r} \cap Y=\emptyset$ could induce an isomorphism. So, $Y$ is smooth and rational and hence so is $X$.

Moreover $Y$ is arithmetically Cohen-Macaulay, so that $H^{2}(B)=0$. In addition $\sigma(X)=$ 0. By Lemma 6.2 we get $H^{2}(A)=0$ and $\sigma(X)=0$.

(b): Let $h=d-r$. Then $Y \subset \mathbb{P}_{K}^{d}$ is of almost minimal degree in the sense of [9]. As $h_{B}^{1}(1)=0$, the surface $Y$ is linearly normal and hence of arithmetic depth $t \geq 2$, (cf. $[9$, Proposition 3.1]).

(i): If $t=3$, then $Y$ is not normal and $Y \backslash \operatorname{Nor}(Y)$ is a line (cf. [9, Theorems 1.4, 1.3]). So, $Y$ is a non-normal maximal Del Pezzo surface in the sense of [9]. Clearly $H^{2}(B)=0$. 
According to $\left[9\right.$, Theorem 6.2] we also have $h_{B}^{3}(-1)=1$ and hence $h_{B}^{3}(0)=0$. So, by Remark 4.8 (D) we get $\sigma(Y)=1$. Now our claims on $X$ follow by Lemma 6.2 (and the fact that $Y$ and $X$ are isomorphic by means of a projection.

(ii): If $t=2$, [9, Theorem 4.2] yields that $h_{B}^{2}(n)=1$ for all $n \leq 0, h_{B}^{2}(n)=0$ for all $n>0$ and $h_{B}^{3}(n)=0$ for all $n \geq-1$. so, by Remark $4.8(\mathrm{~A})$ and (D) we get $e(Y)=1$ and $\sigma(Y)=0$. Moreover, by $[9$, Theorem 1.3] we have $\operatorname{CM}(Y)=\operatorname{Nor}(Y)$. Now we get our claims on $X$ again by Lemma 6.2 and the isomorphism $Y \stackrel{\simeq}{\longrightarrow} X$.

(c): Let $h=d-r-1$. Then $Y \subset \mathbb{P}_{K}^{d-1}$ is of degree $d \geq 6$. Now, on use of Reminder 6.1 (A) and by Lemma 6.2 one easily proves our claims.

\section{A FEW EXAMPLES}

We keep the prevoius notation and hypotheses. The aim of this section is to give a few examples of surfaces which illustrate the results of sections 5 and 6 . If $X \subset \mathbb{P}_{K}^{r}=\operatorname{Proj} S$ with $S=K\left[x_{0}, \ldots, x_{r}\right]$, is a non-degenerate surface with homogeneous vanishing ideal $I$, homogeneous coordinate ring $A$ and arithmetic depth $t$, the Betti diagram of $X$ is the diagram of size $(r-1-t) \times(\operatorname{reg} X-1)$ whose entry in the $i$-th column and the $j$-th row is given by

$$
\beta_{i, j}:=\operatorname{dim}_{k} \operatorname{Tor}_{i}^{S}(K, A)_{i+j}, 1 \leq i \leq r+1-t, 1 \leq j \leq \operatorname{reg} X-1 .
$$

All the occuring computations were performed on use of Singular [14].

We first present an example of a surface $X \subset \mathbb{P}_{K}^{r}$ of degree $d$ which satisfies reg $X=$ $d-r+3$ but is not of maximal sectional regularity (cf. Remark $5.4(\mathrm{~B})$ ). We also show that this surface $X$ has a reduced and irreducible hyperplane section curve $\mathcal{D}$ with $\operatorname{reg} \mathcal{D}=\operatorname{reg} X>\operatorname{sreg} X,($ cf. Remark $5.4(\mathrm{C}))$.

Example 7.1. We project the smooth rational surface scroll $Y:=S(2,5) \subset \mathbb{P}_{K}^{8}$, which is given by the $2 \times 2$-minors of the matrix

$$
\left(\begin{array}{cc|ccccc}
x_{0} & x_{1} & x_{3} & x_{4} & x_{5} & x_{6} & x_{7} \\
x_{1} & x_{2} & x_{4} & x_{5} & x_{6} & x_{7} & x_{8}
\end{array}\right)
$$

from the line $L=\mathbb{P}_{K}^{1} \subset \mathbb{P}_{K}^{8} \backslash \operatorname{Sec}(Y)$ defined by $x_{0}=x_{1}=\ldots=x_{4}=x_{7}=x_{8}=0$. We get a non-degenerate smooth irreducible surface $X \subset \mathbb{P}_{K}^{6}$ of degree 7 which is of arithmetic depth 1 , of regularity 4 and has the Betti diagram

\begin{tabular}{|c|cccccc|}
\hline & 1 & 2 & 3 & 4 & 5 & 6 \\
\hline 1 & 6 & 8 & 3 & 0 & 0 & 0 \\
2 & 4 & 12 & 12 & 4 & 0 & 0 \\
3 & 4 & 18 & 32 & 28 & 12 & 2 \\
\hline
\end{tabular}

Computing $h_{A}^{1}(n)=\operatorname{dim}_{K} \operatorname{Ext}_{S}^{6}(A, S(-7))_{n}$ yields $h_{A}^{1}(n)=2$ for $n=1,2$ and $h_{A}^{1}(n)=0$ for all $n \in \mathbb{Z} \backslash\{1,2\}$. So, the surface $X$ must satisfy sreg $X \in\{3,4\}$ (cf. Reminder 6.1 (B) (ii), (ii)). In particular, we have $2=\delta(X)=$ end $H^{1}(A)=\operatorname{reg} X-2$ (cf. Reminder 6.1 (B) (iii)).

We write $S:=K\left[x_{0}, x_{1}, \ldots, x_{4}, x_{7}, x_{8}\right]$ and $f:=x_{0}-x_{1}-x_{2}-x_{3}-x_{4}-x_{7}-x_{8}$ and consider the hyperplane section $\mathcal{C}=X \cap \operatorname{Proj}(S / f S) \subset \operatorname{Proj}(S / f S)=\mathbb{P}_{K}^{5} \subset \mathbb{P}_{K}^{6}$ of $X$. Computing the primary decomposition of $J:=(I+f S)^{\text {sat }} / f S \subset S / f S=: T$ we find that 
$J \in \operatorname{Spec} T$ so that $\mathcal{C}=\operatorname{Proj}(T / J) \subset \operatorname{Proj} T=\mathbb{P}_{K}^{6}$ is a non-degenerate irreducible curve. The Betti diagram of the curve $Z$ presents itself as follows

\begin{tabular}{|c|ccccc|}
\hline & 1 & 2 & 3 & 4 & 5 \\
\hline 1 & 6 & 8 & 3 & 0 & 0 \\
2 & 6 & 20 & 24 & 12 & 2 \\
\hline
\end{tabular}

In particular the hyperplane section $\mathcal{C}$ of $X$ satisfies $\operatorname{reg} \mathcal{C}=3$. Therefore, sreg $X=3<$ $4=\operatorname{reg} X=\operatorname{deg} X-6+3$. So $X$ is not of maximal sectional regularity, whereas $\operatorname{reg} X$ takes the conjectured maximal value 4 .

Let $X$ and $S$ be as before and let $g:=x_{0}-x_{1}-x_{8}$. Consider the hyperplane section $\mathcal{D}=\operatorname{Proj} A / g A=X \cap \operatorname{Proj} S / g S \subset \operatorname{Proj} S / g S$ of $X$. Again by computing the primary decomposition of $(I+g S)^{\text {sat }} / g S \subset S / g S$ we see that $\mathcal{D} \subset \mathbb{P}_{K}^{5}$ is a non-degenerate reduced and irreducible curve. The Betti diagram of $\mathcal{D}$ is given by

\begin{tabular}{|l|lllll|}
\hline & 1 & 2 & 3 & 4 & 5 \\
\hline 1 & 7 & 8 & 3 & 0 & 0 \\
2 & 0 & 6 & 8 & 3 & 0 \\
3 & 1 & 4 & 6 & 4 & 1 \\
\hline
\end{tabular}

In particular we have indeed $\operatorname{reg} \mathcal{D}=4>\operatorname{sreg} X=3$.

We now give a number of examples which illustrate Theorem 6.3.

Example 7.2. We project the smooth rational surface scroll $Y=S(1,8) \subset \mathbb{P}_{K}^{10}$ given by the $2 \times 2$-minors of the matrix

$$
\left(\begin{array}{c|cccccccc}
x_{0} & x_{2} & x_{3} & x_{4} & x_{5} & x_{6} & x_{7} & x_{8} & x_{9} \\
x_{1} & x_{3} & x_{4} & x_{5} & x_{6} & x_{7} & x_{8} & x_{9} & x_{10}
\end{array}\right)
$$

from the 3 -space $\mathbb{P}_{K}^{3} \subset \mathbb{P}_{K}^{10} \backslash$ Sec $Y$ given by $x_{0}=\ldots=x_{4}=x_{9}=x_{10}=0$. We get a non-degenerate smooth irreducible surface $X \subset \mathbb{P}_{K}^{6}$ of degree 9 which is of arithmetic depth 1, of regularity 6 and has the Betti diagram

\begin{tabular}{|c|cccccc|}
\hline & 1 & 2 & 3 & 4 & 5 & 6 \\
\hline 1 & 6 & 8 & 3 & 0 & 0 & 0 \\
2 & 0 & 0 & 0 & 0 & 0 & 0 \\
3 & 4 & 12 & 12 & 4 & 0 & 0 \\
4 & 4 & 18 & 32 & 28 & 12 & 2 \\
5 & 6 & 28 & 52 & 48 & 22 & 4 \\
\hline
\end{tabular}

A computation of $h_{A}^{1}(n)$ gives the following table for the non-vanishing values of the Hartshorne-Rao function

\begin{tabular}{|c|llll|}
\hline$n$ & 1 & 2 & 3 & 4 \\
\hline$h_{A}^{1}(n)$ & 4 & 8 & 8 & 4 \\
\hline
\end{tabular}

In particular $\delta(X)=3<$ end $H^{1}(A)=4$. Moreover $h_{A}^{1}(1)=4=\operatorname{deg} X-6+1$, so that $X \subset \mathbb{P}_{K}^{6}$ is of type (a) of Theorem 6.3. In accordance with Theorem 6.3 the projecting surface $Y \subset \mathbb{P}_{K}^{9}$ is a smooth rational surface scroll and computing $H^{2}(A) \simeq$ $\operatorname{Hom}_{A}\left(\operatorname{Ext}_{S}^{5}(A, S(-7)), K\right)$ confirms that $H^{2}(A)$ vanishes, as predicted. 
Now, let $f \in S_{1} \backslash\{0\}$. Then, the induced exact sequence

$$
0 \rightarrow H^{0}(A / f A)_{n} \rightarrow H^{1}(A)_{n-1} \stackrel{f}{\rightarrow} H^{1}(A)_{n} \rightarrow H^{1}(A / f A)_{n} \rightarrow 0
$$

and the above table for the values of $h_{A}^{1}(n)$ imply that $h_{A / f A}^{1}(1)=4$ and $h_{A / f A}^{1}(2) \geq 4$. This shows, that statement (c) of Proposition 4.3 need not hold if $d=2 r-3$. So, concerning statement (c), the bound on the degree required in Proposition 4.3 is sharp.

According to Bertini there is a (unique maximal) dense open set $U \subset S_{1} \backslash\{0\}$ such that $\mathcal{C}:=\operatorname{Proj} A / f A=X \cap \operatorname{Proj} S / f S \subset \operatorname{Proj} S / f S=\mathbb{P}_{K}^{5}$ is a non-degenerate reduced irreducible curve of degree 9 for all $f \in U$. If for some $f \in U$ the curve $\mathcal{C}=\operatorname{Proj} A / f A$ is of maximal regularity (that is of regularity 6 ) the inequality $h_{A / f A}^{1}(2) \geq 4>3=\operatorname{deg} \mathcal{C}-5-1$ implies (cf. [7, Theorem 3.3]) that the union of $\mathcal{C}$ with an extremal secant line $\mathbb{L}=\mathbb{P}_{K}^{1}$ of $\mathcal{C}$ is never arithmetically Cohen-Macaulay. We write $S=K\left[x_{0}, \ldots, x_{4}, x_{9}, x_{10}\right]$ and choose $f:=x_{1}-x_{2}$. Then, computing the primary decomposition of $J:=(I+f S)^{\text {sat }} / f S \subset$ $S / f S=: T$ we see that $J \in \operatorname{Spec} T$ so that the hyperplane section $\mathcal{C}=\operatorname{Proj} T / J=$ $X \cap \operatorname{Proj} T \subset \operatorname{Proj} T=\mathbb{P}_{K}^{5}$ is a non-degenerate reduced irreducible curve of degree 9 of arithmetic depth 1 satisfying $\operatorname{reg} \mathcal{C}=6$. The Betti diagram of $\mathcal{C}$ is given by

\begin{tabular}{|c|ccccc|}
\hline & 1 & 2 & 3 & 4 & 5 \\
\hline 1 & 6 & 8 & 3 & 0 & 0 \\
2 & 2 & 4 & 0 & 0 & 0 \\
3 & 1 & 4 & 10 & 6 & 1 \\
4 & 0 & 0 & 0 & 0 & 0 \\
5 & 1 & 4 & 6 & 4 & 1 \\
\hline
\end{tabular}

Clearly $\mathcal{C}$ is a curve of maximal regularity. By the previous observation for each secant line $\mathbb{L}$ of $\mathcal{C}$ the union $\mathcal{C} \cup \mathbb{L}$ is not arithmetically Cohen-Macaulay.

The next two examples shall illustrate the statements of Theorem 6.3 (b) (i) and (ii).

Example 7.3. (A) We start with the same scroll $W=S(1,8) \subset \mathbb{P}_{K}^{10}$ as in the previous example. We project from the point $p=(0: 1: 1: 0: \ldots: 0) \in \mathbb{P}_{K}^{10} \backslash W$ by means of the map $\left(x_{0}: x_{1}: \ldots: x_{10}\right) \mapsto\left(x_{0}: x_{1}-x_{2}: x_{3}: \ldots: x_{10}\right)$. We then get a non-degenerate reduced irreducible surface $Y \subset \mathbb{P}_{K}^{9}$ of degree 9 whose Betti diagram has the shape

\begin{tabular}{|c|ccccccc|}
\hline & 1 & 2 & 3 & 4 & 5 & 6 & 7 \\
\hline 1 & 27 & 105 & 189 & 189 & 105 & 27 & 0 \\
2 & 0 & 0 & 0 & 0 & 0 & 0 & 1 \\
\hline
\end{tabular}

So $Y \subset \mathbb{P}_{K}^{9}$ is a surface of almost minimal degree which is arithmetically Cohen-Macaulay. So $Y$ is maximally Del Pezzo (cf. [9, Theorem 7.2]) and not normal (cf. [9, Theorem 1.3 (a)]). After having introduced new coordinates, we now canonically project $Y \subset \mathbb{P}_{K}^{9}=$ Proj $S, S=K\left[y_{0}, \ldots, y_{9}\right]$, from the plane $\mathbb{P}_{K}^{2} \subset \mathbb{P}_{K}^{9} \backslash Y$ given by $y_{0}=y_{1}=y_{2}=y_{3}=$ $y_{4}=y_{8}=y_{9}=0$. What we get is a non-degenerate reduced irreducible surface $X \subset \mathbb{P}_{K}^{6}$ 
of degree 9 with the following Betti diagram

\begin{tabular}{|c|cccccc|}
\hline & 1 & 2 & 3 & 4 & 5 & 6 \\
\hline 1 & 4 & 2 & 0 & 0 & 0 & 0 \\
2 & 6 & 21 & 20 & 6 & 0 & 0 \\
3 & 0 & 0 & 0 & 0 & 0 & 0 \\
4 & 5 & 23 & 42 & 38 & 17 & 3 \\
\hline
\end{tabular}

So, $X$ is of arithmetic depth 1 and of regularity 5. Moreover, the non-vanishing values of the Hartshorne-Rao function are computed as follows

$$
h_{A}^{1}(1)=3, h_{A}^{1}(2)=4, h_{A}^{1}(3)=3 .
$$

As $h_{A}^{1}(1)=3=\operatorname{deg} X-6$ we are in the situation of statement (b) of Theorem 6.3 and the morphism $Y \rightarrow X$ induced by our projection is an isomorphism. So, $Y$ is a maximal projecting surface for $X$ which is non-normal Del Pezzo. So, we actually are in the case (b) (i) of Theorem 6.3.

As reg $X=5<\operatorname{deg} X-6+3$, the surface $X$ cannot be of maximal sectional regularity (cf. Remark $5.2(\mathrm{~B})$ ). To illustrate this directly, we consider the hyperplane section curve $\mathcal{C}:=\operatorname{Proj} A / f A \subset \operatorname{Proj} S / f S$ with $f:=x_{1}-x_{5}+x_{10}$. Computing the primary decomposition of the vanishing ideal $J \subset S / f S$ of $\mathcal{C}$ we see that this curve is indeed reduced and irreducible. The Betti diagram of $\mathcal{C}$ is computed to be

\begin{tabular}{|c|ccccc|}
\hline & 1 & 2 & 3 & 4 & 5 \\
\hline 1 & 5 & 2 & 0 & 0 & 0 \\
2 & 2 & 15 & 16 & 5 & 0 \\
3 & 0 & 0 & 0 & 0 & 0 \\
4 & 1 & 4 & 6 & 4 & 1 \\
\hline
\end{tabular}

So we have indeed $\operatorname{reg} \mathcal{C}=5<\operatorname{deg} \mathcal{C}-5+2$.

(B) As in part (A) we start with the smooth surface scroll $W=S(1,8) \subset \mathbb{P}_{K}^{10}$. We project $W$ canonically from the point $q=(0: 0: 0: 1: 0: \ldots: 0) \in \mathbb{P}_{K}^{10} \backslash W$ and get again a surface $Y \subset \mathbb{P}_{K}^{9}=\operatorname{Proj} K\left[x_{0}, x_{1}, x_{2}, x_{4}, \ldots, x_{10}\right]$ of degree 9 whose Betti diagram has the shape

\begin{tabular}{|c|cccccccc|}
\hline & 1 & 2 & 3 & 4 & 5 & 6 & 7 & 8 \\
\hline 1 & 26 & 98 & 168 & 154 & 70 & 6 & 0 & 0 \\
2 & 1 & 7 & 21 & 35 & 35 & 28 & 9 & 1 \\
\hline
\end{tabular}

So, $Y$ is a surface of almost minimal degree and arithmetic depth 2 .

We project $Y$ again canonically from the plane $\mathbb{P}_{K}^{2} \subset \mathbb{P}_{K}^{9} \backslash Y$ given by $x_{0}=x_{1}=x_{2}=$ $x_{4}=x_{5}=x_{9}=x_{10}$ and get a surface $X \subset \mathbb{P}_{K}^{6}$ of degree 9 whose Betti diagram is as follows

\begin{tabular}{|c|cccccc|}
\hline & 1 & 2 & 3 & 4 & 5 & 6 \\
\hline 1 & 3 & 2 & 0 & 0 & 0 & 0 \\
2 & 11 & 31 & 30 & 11 & 1 & 0 \\
3 & 0 & 0 & 0 & 0 & 0 & 0 \\
4 & 5 & 23 & 42 & 38 & 17 & 3 \\
\hline
\end{tabular}

In particular $X$ is of arithmetic depth 1 and we have $\operatorname{reg} X=5<\operatorname{deg} X-6+3$. The non-vanishing values of the Hartshorne-Rao function are the same as in the example 
presented in part (A), so that in particular $\delta(X)=2<$ end $H^{1}(A)$ and $h_{A}^{1}(n)=\operatorname{deg} X-6$. The last equation shows again that $Y$ is a projecting surface for $X$ and hence we are in the case of statement (b)(ii) of Theorem 6.3. A computation confirms that $h_{A}^{2}(n)=$ $\operatorname{dim}_{K} \operatorname{Ext}_{S}^{5}(A, S(-7))_{-n}$ equals 1 for all $b \leq 0$ and vanishes for all $n>0$. As reg $X=$ $5<\operatorname{deg} X-6+3$, the surface $X \subset \mathbb{P}_{K}^{6}$ cannot be of maximal sectional regularity (cf. Remark $5.2(\mathrm{~B}))$. This can be confirmed directly by considering the hyperplane section curve $\mathcal{C}=\operatorname{Proj} A / f A \subset \operatorname{Proj} S / f S=\mathbb{P}_{K}^{5}$ with $f=x_{1}-x_{2}$, which is verified to be reduced and irreducible and has the Betti diagram

\begin{tabular}{|c|ccccc|}
\hline & 1 & 2 & 3 & 4 & 5 \\
\hline 1 & 4 & 2 & 0 & 0 & 0 \\
2 & 7 & 25 & 26 & 10 & 1 \\
3 & 0 & 0 & 0 & 0 & 0 \\
4 & 1 & 4 & 6 & 4 & 1 \\
\hline
\end{tabular}

So indeed $\operatorname{reg} \mathcal{C}=5<\operatorname{deg} \mathcal{C}-5+2$. Observe that the necessary condition " $d>2 r-5$ " of Theorem 6.3 is satisfied here. So, the present example shows that (in the case (b) (ii) of Theorem 6.3) this condition is not sufficient to guarantee that $X$ is of maximal sectional regularity.

We now present a few examples which fall under the cases (c) of Theorem 6.3.

Example 7.4. (A) Let $W \subset \mathbb{P}_{K}^{9}$ denote the non-normal Del Pezzo surface considered in Example $7.3(\mathrm{~A})$. We rename our indeterminates and write $\mathbb{P}_{K}^{9}=\operatorname{Proj}\left(K\left[x_{0}, \ldots, x_{9}\right]\right)$. We project $W$ from the point $p:=(1: 0: \ldots: 0: 1)$ by means of the map $\left(x_{0}: \ldots: x_{9}\right) \mapsto$ $\left(x_{0}-x_{9}: x_{1}: \ldots: x_{8}\right)$ and get a surface $Y \subset \mathbb{P}_{K}^{8}$ of degree 9 whose Betti diagram is

\begin{tabular}{|c|cccccc|}
\hline & 1 & 2 & 3 & 4 & 5 & 6 \\
\hline 1 & 19 & 58 & 75 & 44 & 5 & 0 \\
2 & 0 & 0 & 0 & 0 & 6 & 2 \\
\hline
\end{tabular}

In particular $Y$ now is arithmetically Cohen-Macaulay. Moreover the canonical module $K(A)=K^{3}(A)=\operatorname{Ext}_{S}^{6}(A, S(-9))$ is computed to be minimally generated by 2 homogeneous elements of degree 1. So, in particular $Y$ must be of type I (cf. Reminder 6.1 (A)).

Now, we project $Y$ from the line $\mathbb{P}_{K}^{1} \subset \mathbb{P}_{K}^{8} \backslash Y$ given by $x_{0}=x_{1}=x_{2}=x_{3}=x_{6}=$ $x_{7}=x_{8}=0$ and get a surface $X \subset \mathbb{P}_{K}^{6}$ of degree 9 with the diagram

\begin{tabular}{|c|cccccc|}
\hline & 1 & 2 & 3 & 4 & 5 & 6 \\
\hline 1 & 3 & 2 & 0 & 0 & 0 & 0 \\
2 & 13 & 39 & 42 & 19 & 3 & 0 \\
3 & 1 & 5 & 10 & 10 & 5 & 1 \\
\hline
\end{tabular}

The non-vanishing values of the Hartshorne-Rao function are computed to be $h_{A}^{1}(1)=$ $2, h_{A}^{1}(2)=2$ and $H^{2}(A)$ turns out to be 0 . So, we must be in the situation described in statement (c) (i) of Theorem 6.3. Again we have $\delta(X)=$ end $H^{1}(A)=2<6=$ $\operatorname{deg} X-6+3$ and $\operatorname{reg} X=4$ so that $X$ surely cannot be of maximal sectional regularity.

Indeed, by computation it turns out that the hyperplane section curve $\mathcal{C}=\operatorname{Proj} A / f A$ of $X$ is reduced and irreducible for $f=x_{0}-x_{6}$ and $f=x_{1}-x_{2}$. It is interesting to note 
that in the first case $\operatorname{reg} \mathcal{C}$ takes the maximally possible value $4=\operatorname{reg} X$, where as in the second case we have $\operatorname{reg} \mathcal{C}=3$.

(B) Our next example is of the type mentioned under statement 6.3 (c) (ii). We first project the scroll $W=S(3,6) \subset \mathbb{P}_{K}^{10}=\operatorname{Proj} K\left[x_{0}, \ldots, x_{10}\right]$ given by the $2 \times 2$-minors of the matrix

$$
\left(\begin{array}{lll|llllll}
x_{0} & x_{1} & x_{2} & x_{4} & x_{5} & x_{6} & x_{7} & x_{8} & x_{9} \\
x_{1} & x_{2} & x_{3} & x_{5} & x_{6} & x_{7} & x_{8} & x_{9} & x_{10}
\end{array}\right)
$$

canonically from the line $\mathbb{P}_{K}^{1} \subset \mathbb{P}_{K}^{10} \backslash W$ defined by $x_{0}=x_{1}=x_{3}=x_{4}=\ldots=x_{8}=$ $x_{10}=0$. We get a surface $Y \subset \mathbb{P}_{K}^{8}=\operatorname{Proj}\left(K\left[x_{0}, x_{1}, x_{3}, \ldots, x_{8}, x_{10}\right]\right)$ of degree 9 , arithmetic depth 2, regularity 3 and having the Betti diagram

\begin{tabular}{|c|ccccccc|}
\hline & 1 & 2 & 3 & 4 & 5 & 6 & 7 \\
\hline 1 & 18 & 52 & 60 & 24 & 0 & 0 & 0 \\
2 & 1 & 6 & 15 & 30 & 27 & 9 & 1 \\
\hline
\end{tabular}

In particular $Y$ can only be of type IIA, IIA' or III A (cf. Reminder 6.1 (A)). We now project $Y$ canonically from the line $\mathbb{P}_{K}^{1} \subset \mathbb{P}_{K}^{8} \backslash Y$ given by $x_{0}-x_{8}=x_{1}=x_{3}=x_{4}=x_{5}=$ $x_{7}=x_{10}$. We obtain a surface $X \subset \mathbb{P}_{K}^{6}$ of degree 9 with Betti diagram

\begin{tabular}{|c|cccccc|}
\hline & 1 & 2 & 3 & 4 & 5 & 6 \\
\hline 1 & 3 & 0 & 0 & 0 & 0 & 0 \\
2 & 9 & 30 & 27 & 8 & 0 & 0 \\
3 & 3 & 15 & 29 & 27 & 12 & 2 \\
\hline
\end{tabular}

In particular $X$ is of arithmetic depth 1 and satisfies $\operatorname{reg} X=4$. The non-vanishing values of the Hartshorne-Rao function of $X$ are computed to be $h_{A}^{1}(n)=2$ for $n=1,2$. In particular $h_{A}^{1}(1)=2=\operatorname{deg} X-6-1$, so that $Y \subset \mathbb{P}_{K}^{8}$ is a maximal projecting surface for $X$ and we must be in one of the cases mentioned in statement (c) of Theorem 6.3. Another computation yields that $h_{A}^{2}(0)=1$ and $h_{A}^{2}(n)=0$ for all $n \neq 0$. So, we must be in the case (c) (ii). If $f \in S_{1} \backslash\{0\}$ is a linear form such that the hyperplane section curve $\mathcal{C}=\operatorname{Proj} A / f A$ of $X$ is reduced and irreducible, we must have $3 \leq \operatorname{reg} \mathcal{C} \leq 4$. Choosing $f:=x_{3}-x_{4}$ we get indeed a reduced irreducible hyperplane section curve $\mathcal{C}$ of regularity 4 .

(C) Next, we present an example for the case (c) (iii) of Theorem 6.3. Again we start with the scroll $W=S(1,8) \subset \mathbb{P}_{K}^{10}$ and project $W$ from the line $\mathbb{P}_{K}^{1} \subset \mathbb{P}_{K}^{10} \backslash W$ given by $x_{0}=x_{1}-x_{2}=x_{3}=x_{5}=\ldots=x_{10}=0$. We thus get a surface $Y \subset \mathbb{P}_{K}^{8}$ of degree nine whose Betti diagram is the same as the Betti diagram of the surface $Y$ in part (B).

So, the homogeneous coordinate ring $B$ of $Y$ has depth 2. Moreover, $H^{2}(B)$ is calculated to be isomorphic to the Matlis dual of $K\left[x_{0}\right]$. So, $Y$ falls under the type IIA' mentioned in Reminder 6.1 (A) (iii). Now, we project $Y$ from the point $(0: \ldots: 0: 1: 0: 0) \in \mathbb{P}_{K}^{8} \backslash Y$ and get a surface $X \subset \mathbb{P}_{K}^{7}$ of degree 9 with Betti diagram

\begin{tabular}{|c|ccccccc|}
\hline & 1 & 2 & 3 & 4 & 5 & 6 & 7 \\
\hline 1 & 9 & 11 & 0 & 0 & 0 & 0 & 0 \\
2 & 5 & 36 & 81 & 75 & 36 & 9 & 1 \\
\hline
\end{tabular}

whose homogeneous coordinate ring $A$ satisfies $H^{1}(A) \simeq K(-1)$. Now clearly we are in the requested case with $d=9, r=7$ and $h=1$. As sreg $Y=3$ by Reminder 6.1 (A) 
(iii) we must have sreg $X \geq 3$ (cf. Lemma $6.2(\mathrm{e})$ ). For various linear forms, for instance $f=x_{0}-x_{1}-x_{5}-x_{6}$, we computed $\operatorname{reg} \operatorname{Proj}(A / f A)=3$, whence sreg $X=3$.

(D) We now present an example for the case (c) (iv) of Theorem 6.3. We first project the scroll $W=S(3,6) \subset \mathbb{P}_{K}^{10}$ of part $(\mathrm{B})$ from the line $\mathbb{P}_{K}^{1} \subset \mathbb{P}_{K}^{10} \backslash W$ given by $x_{0}=x_{2}=$ $\ldots=x_{8}=x_{10}=0$. We then get a surface $Y \subset \mathbb{P}_{K}^{8}=\operatorname{Proj}\left(K\left[x_{0}, x_{2}, \ldots, x_{8}, x_{10}\right]\right)$ of degree 9 whose Betti diagram is given by

\begin{tabular}{|c|ccccccc|}
\hline & 1 & 2 & 3 & 4 & 5 & 6 & 7 \\
\hline 1 & 17 & 46 & 45 & 8 & 0 & 0 & 0 \\
2 & 2 & 12 & 34 & 65 & 48 & 16 & 2 \\
\hline
\end{tabular}

In particular, $Y$ is of arithmetic depth 2 and satisfies $\operatorname{reg} Y=3$. So $Y$ must be of type IIA, IIA' or IIIA (cf. Reminder $6.1(\mathrm{~A})$ ). We now project $Y$ canonically from the point $p=(0: 0: 0: 0: 0: 1: 0: 0: 0) \in \mathbb{P}_{K}^{8} \backslash Y$ and get a surface $X \subset \mathbb{P}_{K}^{7}$ of degree 9 and regularity 3 with Betti diagram

\begin{tabular}{|c|ccccccc|}
\hline & 1 & 2 & 3 & 4 & 5 & 6 & 7 \\
\hline 1 & 8 & 12 & 3 & 0 & 0 & 0 & 0 \\
2 & 12 & 54 & 101 & 90 & 42 & 10 & 1 \\
\hline
\end{tabular}

In particular, $X$ is of arithmetic depth 1 . Moreover by computation we get $H^{1}(A) \simeq$ $K(-1)$ and $h_{A}^{2}(n)=2$ for all $n \leq 0$. This shows that $Y$ is a maximal projecting surface for $X$ and that we are in the case (c)(iv) of Theorem 6.3. The intersection curve $\mathcal{C}=$ $\operatorname{Proj}\left(A /\left(x_{0}-x_{10}\right) A\right)$ is computed to be reduced and irreducible and to satisfy reg $\mathcal{C}=3$. As $X$ is of arithmetic depth 1, each hyperplane section of $X$ must be of regularity $\geq 3$. So, the generic hyperplane section of $X$ must be a curve of regularity 3. In particular $X$ cannot be of maximal sectional regularity.

(E) Finally we present an example for the case (c) (v) of Theorem 6.3. This time we project the scroll $W=S(1,8) \subset \mathbb{P}_{K}^{10}$ from the line $\mathbb{P}_{K}^{1} \subset \mathbb{P}^{10} \backslash W$ given by $x_{0}=x_{1}=x_{2}=$ $x_{5}=\ldots=x_{10}=0$ and get a surface $Y \subset \mathbb{P}_{K}^{8}$ of degree 9 with Betti diagram

\begin{tabular}{|c|ccccccc|}
\hline & 1 & 2 & 3 & 4 & 5 & 6 & 7 \\
\hline 1 & 18 & 52 & 60 & 24 & 5 & 0 & 0 \\
2 & 0 & 0 & 0 & 15 & 12 & 3 & 0 \\
3 & 1 & 6 & 15 & 20 & 15 & 6 & 1 \\
\hline
\end{tabular}

So the homogeneous coordinate ring $B$ of $Y$ has depth 2. Moreover a computation furnishes that $H^{2}(B) \simeq\left(K\left[x_{0}, x_{1}, x_{2}\right] /\left(x_{0}, x_{1}\right)^{2}\right)(-1)$. So, $Y$ must be of type IVA1 of Reminder $6.1(\mathrm{~A})(\mathrm{v})$. Now, we project $Y$ from the point $(0: \ldots: 0: 1: 1: 0: 0) \in \mathbb{P}_{K}^{8} \backslash Y$, and get a surface $X \subset \mathbb{P}_{K}^{7}$ of degree nine with Betti diagram

\begin{tabular}{|c|ccccccc|}
\hline & 1 & 2 & 3 & 4 & 5 & 6 & 7 \\
\hline 1 & 9 & 12 & 3 & 0 & 0 & 0 & 0 \\
2 & 5 & 34 & 71 & 65 & 31 & 8 & 1 \\
3 & 1 & 5 & 10 & 10 & 5 & 1 & 0 \\
\hline
\end{tabular}

In particular the homogeneous coordinate ring $A$ of $X$ has depth 1 , and a further computation shows that $H^{1}(A) \simeq K(-1)$. So, this time we are in the case (c) (v) of Theorem 6.3 with $d=9, r=7$ and $h=1$. Again by Lemma $6.2(\mathrm{e})$, Reminder $6.1(\mathrm{~A})(\mathrm{v})$ and by 
computing reg $\operatorname{Proj}(A / f A)$ for some linear forms $f$, e.g. $f=x_{0}-x_{2}+x_{10}$, we obtain that sreg $X=4$.

We now return to the surface $Y \subset \mathbb{P}_{K}^{8}$. The Betti diagram of $Y$ tells us, that the homogeneous vanishing ideal $J \subset S=K\left[x_{0}, x_{1}, x_{2}, x_{5}, \ldots, x_{10}\right]$ of $Y$ is generated by 18 quadrics and one quartic $Q$. A Singular computation gives $Q=x_{1}^{3} x_{2}-x_{0}^{3} x_{5}$ and $L:=$ $J_{2} S:_{S} Q=\left(x_{5}, x_{6}, x_{7}, x_{8}, x_{9}, x_{10}\right)$. So $\mathbb{E}:=\operatorname{Proj} S / L=\mathbb{P}_{K}^{2}$ is a plane and $J+L=L+Q S$ tells us that $Y \cap \mathbb{E}$ is the quartic defined in $\mathbb{E}$ by $Q$. So, $Y$ admits a whole plane of $4=(\operatorname{deg} Y-8+3)$-secant lines, which is in accordance with the fact that $Y$ is of maximal sectional regularity (cf. Corollaries 5.6 and 5.7).

Remark 7.5. The Singular files of the examples in this section are available upon request by the authors.

\section{REFERENCES}

[1] Bertin, M. A.: On the regularity of varieties having an extremal secant line, J. Reine Angewand. Math. 545 (2002), 167-181.

[2] Bertin, M. A.: On singular varieties having an extremal secant line, Comm. Algebra 34 (2006), 893-909.

[3] Brodmann, M.: Bounds on the cohomological Hilbert functions of a projective variety, J. Algebra 109 (1987), 352-380.

[4] Brodmann, M.: Cohomology of certain projective surfaces with low sectional genus and degree, in: D. Eisenbud (Ed.) "Commutative Algebra, Algebraic Geometry and Computational Methods", 172 - 200, Springer, 1999.

[5] Brodmann, M.: Cohomology of surfaces $X \subset \mathbb{P}_{K}^{r}$ with degree $\leq 2 r-2$, in: F. van Oystayen (Ed.) "Commutative Algebra and Algebraic Geometry", 15 - 33, M. Dekker, Lect. Notes 2006, 1999.

[6] Brodmann, M., Schenzel, P.: Curves of degree $r+2$ in $\mathbb{P}^{r}$ : Cohomological, geometric, and homological aspects, J. Algebra 242 (2001), 577 - 623.

[7] Brodmann, M., Schenzel, P.: On projective curves of maximal regularity, Math. Z. 244 (2003), $271-289$.

[8] Brodmann, M., Schenzel, P.: On varieties of almost minimal degree in small codimension, J. Algebra 305 (2006), $789-801$.

[9] Brodmann, M., Schenzel, P.: Arithmetic properties of projective varieties of almost minimal degree, J. Algebraic Geom. 16 (2007), 347 - 400.

[10] Eisenbud, D., GôTo, S.: Linear free resolutions and minimal multiplicity, J. Algebra 88 (1984), 89-133.

[11] Fujita, T.: "Classification theories of polarized varieties", London Math. Soc., Lect. Notes Ser. 155, Cambridge Univ. Press, 1990.

[12] Green, M.: Koszul homology and the geometry of projective varieties, J. Differential Geometry 19 (1984), 125 - 171.

[13] Green, M., Lazarsfeld, R.: Some results on the syzygies of finite sets and algebraic curves. Compositio Math. 67 (1988), 301 - 314.

[14] Greuel, G.M., Pfister, G. ET AL: Singular 3.0.4, a computer algebra system for polynomial computations. Center for Computer Algebra, University of Kaiserslautern (2007) (http://www.singular.uni-kl.de).

[15] Gruson, L., Lazarsfeld, R., Peskine, C.: On a theorem of Castelnuovo and the equations defining space curves, Inventiones Math. 72 (1983), 491-506.

[16] HARRIS, J.: "Algebraic Geometry: A first course", Graduate Texts in Math., Vol. 133, Springer, New-York, 1992.

[17] Huneke, C., Ulrich, B.: General hyperplane sections of algebraic varieties, J. Algebraic Geom. 2 (1993), $487-505$. 
[18] Kreuzer, M.: An introduction to a Theorem of Strano, in: The Curves Seminar at Queen's, Vol. VIII, Queen's Papers in Pure and Appl. Math. 88, Queen's University, Kingston 1991.

[19] Mumford, D.: "Lectures on curves on an algebraic surface", Ann. Math. Studies 59, Princeton Univ. Press, 1966.

Universität Zürich, Institut für Mathematik, Winterthurer Str. 190, CH - 8057 ZÜrICH, SCHWITZERLAND

E-mail address: markus.brodmann@math.uzh.ch

Martin-Luther-Universität Halle-Wittenberg, Institut für Informatik, D - 06099 Halle (SaAle), Germany

E-mail address: peter.schenzel@informatik.uni-halle.de 